\title{
The Uncanny Precision of the Spectral Action
}

\author{
Ali H. Chamseddine ${ }^{1,3}$, Alain Connes Co, $^{2,3}$ \\ ${ }^{1}$ Physics Department, American University of Beirut, Lebanon \\ ${ }^{2}$ College de France, 3 rue Ulm, F75005, Paris, France \\ ${ }^{3}$ I.H.E.S. F-91440 Bures-sur-Yvette, France \\ ${ }^{4}$ Department of Mathematics, Vanderbilt University, Nashville, TN 37240 USA
}

\begin{abstract}
Noncommutative geometry has been slowly emerging as a new paradigm of geometry which starts from quantum mechanics. One of its key features is that the new geometry is spectral in agreement with the physical way of measuring distances. In this paper we present a detailed introduction with an overview on the study of the quantum nature of space-time using the tools of noncommutative geometry. In particular we examine the suitability of using the spectral action as action functional for the theory. To demonstrate how the spectral action encodes the dynamics of gravity we examine the accuracy of the approximation of the spectral action by its asymptotic expansion in the case of the round sphere $S^{3}$. We find that the two terms corresponding to the cosmological constant and the scalar curvature term already give the full result with remarkable accuracy. This is then applied to the physically relevant case of $S^{3} \times S^{1}$ where we show that the spectral action in this case is also given, for any test function, by the sum of two terms up to an astronomically small correction, and in particular all higher order terms $a_{2 n}$ vanish. This result is confirmed by evaluating the spectral action using the heat kernel expansion where we check that the higher order terms $a_{4}$ and $a_{6}$ both vanish due to remarkable cancelations. We also show that the Higgs potential appears as an exact perturbation when the test function used is a smooth cutoff function.
\end{abstract}




\section{AN OVERVIEW}

Our experimental information on the nature of space-time is based on two sources:

- High energy physics based on cosmic ray information and particle accelerator experiments, whose results are encapsulated in the Standard Model of particle physics.

- Cosmology based on astronomical observations.

The large scale global picture is well described in terms of Riemannian geometry and general relativity, but this picture breaks down at high energy where the quantum effects take over. It is thus natural to look for a paradigm of geometry which starts from the quantum framework, where the role of real variables is played by self-adjoint operators in Hilbert space. Such a framework for geometry has been slowly emerging under the name of noncommutative geometry. One of its key features, besides the ability to handle spaces for which coordinates no longer commute with each other, is that this new geometry is spectral. This is in agreement with physics in which most of the data we have, either about the far distant parts of the universe or about high energy physics, are also of spectral nature. The red shifted spectra of distant galaxies or the momentum eigenstates of outgoing particles in high energy experiments both point towards a prevalence of spectral information. In the same vein the existing unit of time (length) is also of spectral nature. From the mathematical standpoint it takes some doing to obtain a purely spectral (Hilbert space theoretical) counterpart of Riemannian geometry. One reason for the difficulty of this task is that, as is well known since the examples of J. Milnor [1, non-isometric Riemannian spaces exist which have the same spectra (for the Dirac or Laplacian operators). Another reason is that the conditions for a (compact) space to be a smooth manifold are given in terms of the local charts, whose existence and compatibility is assumed, but whose intrinsic meaning is more elusive.

The paradigm of noncommutative geometry is that of spectral triple. As its name indicates it is of spectral nature. By definition a spectral triple is a unitary Hilbert space representation of "something". This something is an equipment that allows one to manipulate algebraically coordinates and to measure distances. The algebra of the coordinates is denoted by $\mathcal{A}$ and is an involutive algebra, with involution $a \mapsto a^{*}$. The equipment needed to measure distances is the inverse line element $D$ which is unbounded and fulfills $D=D^{*}$. Altogether these data fulfill some algebraic relations, e.g. if we talk about the simplest geometric space i.e. the circle $S^{1}$ the relation between the complex unitary coordinate $U$ and the inverse line element $D$ is just $[D, U]=U$, which is in the vein of the Heisenberg commutation relations.

Thus, a geometry is given as a Hilbert space representation of the pair $(\mathcal{A}, D)$ and can be encoded by the spectral triple $(\mathcal{A}, \mathcal{H}, D)$ where $\mathcal{H}$ is the Hilbert space in which both the algebra $\mathcal{A}$ and the inverse line element $D$ are now 
concretely represented, the latter as an unbounded self-adjoint operator. This picture shares with the Wigner paradigm for a particle as an (irreducible) representation of the Poincaré group the feature that it separates the kinematical relations from the choice of the Hilbert space representation. It is only when the latter is chosen that actual measurements of distances between points $x$ and $y$ can be performed by formulas such as

$$
\text { Distance }(x, y)=\sup |f(x)-f(y)|, f \in \mathcal{A},\|[D, f]\| \leq 1
$$

where indeed the norm $\|[D, f]\|$ is the operator norm in Hilbert space and depends on the specific choice of the representation.

We now have at our disposal a reconstruction theorem (cf [2]) which shows that ordinary Riemannian spaces are neatly characterized among spectral triples by the following kinematical relations:

- The algebra $\mathcal{A}$ is commutative.

- The commutator $[[D, a], b]=0$ for any $a, b \in \mathcal{A}$.

- The following "Heisenberg type" relation 1 holds 2 , for some $a_{j}^{\alpha} \in \mathcal{A}$ :

$$
\sum_{\alpha} a_{0}^{\alpha}\left[\left[D, a_{1}^{\alpha}\right],\left[D, a_{2}^{\alpha}\right], \ldots,\left[D, a_{n}^{\alpha}\right]\right]=1
$$

together with the following spectral requirements:

- The $k$-th characteristic value of the resolvent of $D$ is $O\left(k^{-1 / n}\right)$.

- Regularity.

- Absolute continuity.

We refer to [2] for the precise statement. The meaning of (1) is that the determinant of the metric $g^{\mu \nu}$ does not vanish, and more precisely that its square root multiplied by the volume form $\sum_{\alpha} a_{0}^{\alpha} d a_{1}^{\alpha} \wedge d a_{2}^{\alpha} \wedge \cdots d a_{n}^{\alpha}$ gives 1 . The reason for the last two spectral requirements is technical and allows one to specify the regularity $\left(C^{\infty}\right.$, real analytic...) of the space and to control the spectral measures. The first of the spectral requirements is crucial in that it bounds the "effective dimension" of the spectrum of the space in the representation. There are good physics reasons to consider that the apparent dimension, equal to four, of space-time is governed by the asymptotic behavior of the eigenvalues of the line element, which is the Euclidean propagator. Moreover this spectral dimension is not restricted to be an integer a priori and can model fractal dimension easily. The above reconstruction Theorem shows furthermore that the operator $D$ in the spectral triple is a Dirac type operator, i.e. an order one operator with symbol given by a representation of the Clifford algebra. The restriction to spin manifolds is obtained by requiring a real structure i.e. an antilinear unitary operator $J$

\footnotetext{
${ }^{1}$ Here the multiple commutator is defined as

$$
\left[T_{1}, T_{2}, \ldots, T_{n}\right]=\sum_{\sigma} \epsilon(\sigma) T_{\sigma(1)} T_{\sigma(2)} \cdots T_{\sigma(n)}
$$
}

${ }^{2}$ We assume for simplicity that the dimension $n$ is odd 
acting in $\mathcal{H}$ which plays the same role and has the same algebraic properties as the charge conjugation operator in physics. When the dimension $n$ involved in the reconstruction Theorem is even (rather than odd) the right hand side of (10) is now replaced by the chirality operator $\gamma$ which is just a $\mathbb{Z} / 2$-grading in mathematical terms. It fulfills the rules

$$
\gamma^{2}=1, \quad[\gamma, a]=0, \quad a \in \mathcal{A}
$$

The following further relations hold for $D, J$ and $\gamma$

$$
J^{2}=\varepsilon, D J=\varepsilon^{\prime} J D, \quad J \gamma=\varepsilon^{\prime \prime} \gamma J, \quad D \gamma=-\gamma D
$$

where $\varepsilon, \varepsilon^{\prime}, \varepsilon^{\prime \prime} \in\{-1,1\}$. The values of the three signs $\varepsilon, \varepsilon^{\prime}, \varepsilon^{\prime \prime}$ depend only, in the classical case of spin manifolds, upon the value of the dimension $n$ modulo 8 and are given in the following table [3]:

\begin{tabular}{|c|rrrrrrrr|}
\hline $\mathbf{n}$ & 0 & 1 & 2 & 3 & 4 & 5 & 6 & 7 \\
\hline \hline$\varepsilon$ & 1 & 1 & -1 & -1 & -1 & -1 & 1 & 1 \\
$\varepsilon^{\prime}$ & 1 & -1 & 1 & 1 & 1 & -1 & 1 & 1 \\
$\varepsilon^{\prime \prime}$ & 1 & & -1 & & 1 & & -1 & \\
\hline
\end{tabular}

In the classical case of spin manifolds there is thus a relation between the metric (or spectral) dimension given by the rate of growth of the spectrum of $D$ and the integer modulo 8 which appears in the above table. For more general spaces however the two notions of dimension (the dimension modulo 8 is called the $K O$-dimension because of its origin in $K$-theory) become independent since there are spaces $F$ of metric dimension 0 but of arbitrary $K O$-dimension. More precisely, starting with an ordinary spin geometry $M$ of dimension $n$ and taking the product $M \times F$, one obtains a space whose metric dimension is still $n$ but whose $K O$-dimension is the sum of $n$ with the $K O$-dimension of $F$, which as explained can take any value modulo 8 . Thus, one now has the freedom to shift the $K O$-dimension at very little expense i.e. in a way which does not alter the plain metric dimension. As it turns out the Standard Model with neutrino mixing favors the shift of dimension from the 4 of our familiar space-time picture to $10=4+6=2$ modulo 8 [4], [5]. The shift from 4 to 10 is a recurrent idea in string theory compactifications, where the 6 is the dimension of the Calabi-Yau manifold used to "compactify". Effectively the dimension 10 is related to the existence of Majorana-Weyl fermions. The difference between this approach and ours is that, in the string compactifications, the metric dimension of the full space-time is now 10 which can only be reconciled with what we experience by requiring that the Calabi-Yau fiber remains unnaturally small. In order to learn how to perform the above shift of dimension using a 0 -dimensional space $F$, it is important to classify such spaces. This was done in [6], 7]. There, we classified the finite spaces $F$ of given $K O$-dimension. A space $F$ is finite when the algebra $\mathcal{A}_{F}$ of coordinates on $F$ is finite dimensional. We no longer require that this algebra is commutative. The first key advantage 
of dropping the commutativity can be seen in the simplest case where the finite space $F$ is given by

$$
\mathcal{A}=M_{k}(\mathbb{C}), \mathcal{H}=M_{k}(\mathbb{C}), D=0, J \xi=\xi^{*}, \quad \xi \in \mathcal{H}_{F}
$$

where the algebra $\mathcal{A}=M_{k}(\mathbb{C})$ is acting by left multiplication in $\mathcal{H}=M_{k}(\mathbb{C})$. We have shown in $[8$ that the study of pure gravity on the space $M \times F$ yields Einstein gravity on $M$ minimally coupled with Yang-Mills theory for the gauge group $\mathrm{SU}(k)$. The Yang-Mills gauge potential appears as the inner part of the metric, in the same way as the group of gauge transformations (for the gauge group $\mathrm{SU}(k)$ ) appears as the group of inner diffeomorphisms. One can see in this Einstein-Yang-Mills example that the finite geometry fulfills a nice substitute of commutativity (of $\mathcal{A}$ ) namely

$$
\left[a, b^{0}\right]=0, \quad \forall a, b \in \mathcal{A}
$$

where for any operator $a$ in $\mathcal{H}, a^{0}=J a^{*} J^{-1}$. This is called the order zero condition. Moreover the representation of $\mathcal{A}$ and $J$ in $\mathcal{H}$ is irreducible. This example is (taking $\gamma=1$ ) of $K O$-dimension equal to 0 . In [6] we classified the irreducible $(\mathcal{A}, \mathcal{H}, J)$ and found out that the solutions fall into two classes. Let $\mathcal{A}_{\mathbb{C}}$ be the complex linear space generated by $\mathcal{A}$ in $\mathcal{L}(\mathcal{H})$, the algebra of operators in $\mathcal{H}$. By construction $\mathcal{A}_{\mathbb{C}}$ is a complex algebra and one only has two cases:

(1) The center $Z\left(\mathcal{A}_{\mathbb{C}}\right)$ is $\mathbb{C}$, in which case $\mathcal{A}_{\mathbb{C}}=M_{k}(\mathbb{C})$ for some $k$.

(2) The center $Z\left(\mathcal{A}_{\mathbb{C}}\right)$ is $\mathbb{C} \oplus \mathbb{C}$ and $\mathcal{A}_{\mathbb{C}}=M_{k}(\mathbb{C}) \oplus M_{k}(\mathbb{C})$ for some $k$. Moreover the knowledge of $\mathcal{A}_{\mathbb{C}}=M_{k}(\mathbb{C})$ shows that $\mathcal{A}$ is either $M_{k}(\mathbb{C})$ (unitary case), $M_{k}(\mathbb{R})$ (real case) or, when $k=2 \ell$ is even, $M_{\ell}(\mathbb{H})$, where $\mathbb{H}$ is the field of quaternions (symplectic case). This first case is a minor variant of the Einstein-Yang-Mills case described above. It turns out by studying their $\mathbb{Z} / 2$ gradings $\gamma$, that these cases are incompatible with $K O$ dimension 6 which is only possible in case (2). If one assumes that one is in the "symplectic-unitary" case and that the grading is given by a grading of the vector space over $\mathbb{H}$, one can show that the dimension of $\mathcal{H}$ which is $2 k^{2}$ in case (2) is at least $2 \times 16$ while the simplest solution is given by the algebra $\mathcal{A}=M_{2}(\mathbb{H}) \oplus M_{4}(\mathbb{C})$. This is an important variant of the Einstein-YangMills case because, as the center $Z\left(\mathcal{A}_{\mathbb{C}}\right)$ is $\mathbb{C} \oplus \mathbb{C}$, the product of this finite geometry $F$ by a manifold $M$ appears, from the commutative standpoint, as two distinct copies of $M$. We showed in [6] that requiring that these two copies of $M$ stay a finite distance apart reduces the symmetries from the group $\mathrm{SU}(2) \times \mathrm{SU}(2) \times \mathrm{SU}(4)$ of inner automorphism 3 to the symmetries $U(1) \times \mathrm{SU}(2) \times \mathrm{SU}(3)$ of the Standard Model. This reduction of the gauge symmetry occurs because of the second kinematical condition $[[D, a], b]=0$ which in the general case becomes:

$$
\left[[D, a], b^{0}\right]=0, \quad \forall a, b \in \mathcal{A}
$$

\footnotetext{
3 of the even part of the algebra
} 


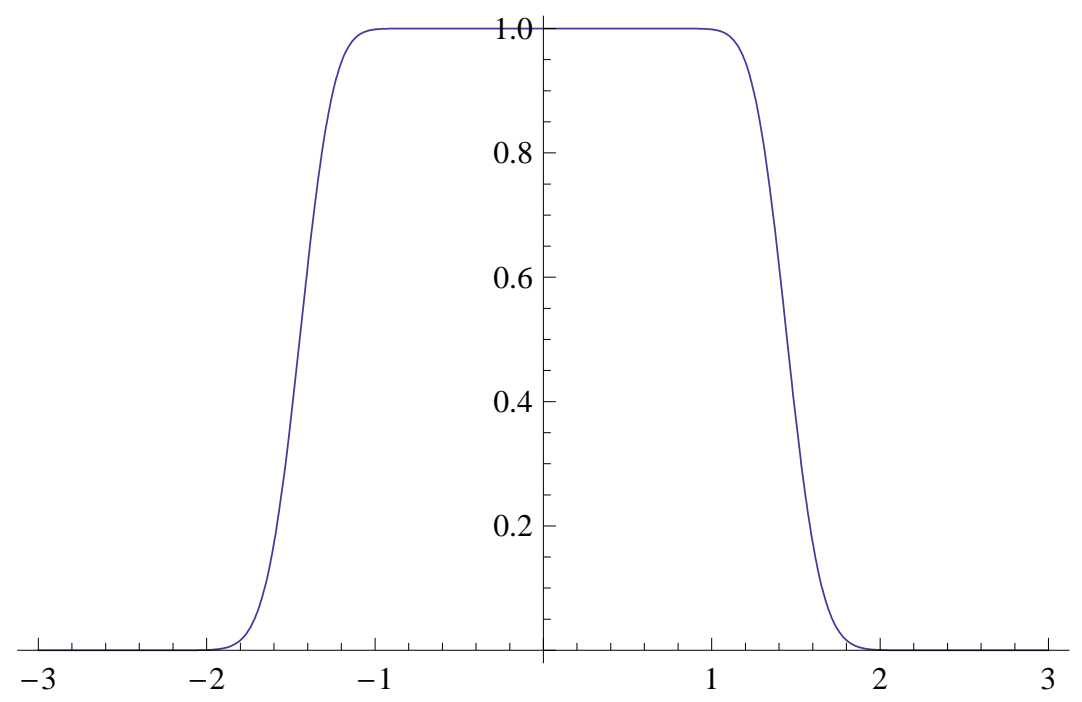

Figure 1. Cutoff function $\mathrm{f}$

Thus the noncommutative space singles out $4^{2}=16$ as the number of physical fermions, the symmetries of the standard model emerge, and moreover, as shown in [9], the model predicts the existence of right-handed neutrinos, as well as the see-saw mechanism. In the above Einstein-Yang-Mills case, the Yang-Mills fields appeared as the inner part of the metric in the same way as the group of gauge transformations (for the gauge group $\mathrm{SU}(k)$ ) appeared as the group of inner diffeomorphisms. But in that case all fields remained massless. It is the existence of a non-zero $D$ for the finite space $F$ that generates the Higgs fields and the masses of the Fermions and the $W$ and $Z$ fields through the Higgs mechanism. The new fields are computed from the kinematics but the action functional, the spectral action, uses in a crucial manner the representation in Hilbert space. In order to explain the conceptual meaning of this spectral action functional it is important to understand in which way it encodes gravity in the commutative case. As explained above the spectrum of the Dirac operator (or similarly of the Laplacian) does not suffice to encode an ordinary Riemannian geometry. However the Einstein-Hilbert action functional, given by the integral of the scalar curvature multiplied by the volume form, appears from the heat expansion of the Dirac operator. More generally it appears as the coefficient of $\Lambda^{2}$ in the asymptotic expansion for large $\Lambda$ of the trace

$$
\operatorname{Tr}(f(D / \Lambda)) \sim 2 \Lambda^{4} f_{4} a_{0}+2 \Lambda^{2} f_{2} a_{2}+f_{0} a_{4}+\ldots+\Lambda^{-2 k} f_{-2 k} a_{4+2 k}+\ldots
$$

when the Riemannian geometry $M$ is of dimension 4, and where $f$ is a smooth even function with fast decay at infinity. The choice of the function $f$ only enters in the multiplicative factors $f_{4}=\int_{0}^{\infty} f(u) u^{3} d u$, 
$f_{2}=\int_{0}^{\infty} f(u) u d u, f_{0}=f(0)$ and $f_{-2 k}=(-1)^{k} \frac{k !}{(2 k) !} f^{(2 k)}(0)$, i.e. the derivatives of even order at 0 , for $k \geq 0$. Thus, when $f$ is a "cutoff" function (cf Figure 10 it has vanishing Taylor expansion at 0 and the asymptotic expansion (7) only has three terms:

$$
\operatorname{Tr}(f(D / \Lambda)) \sim 2 \Lambda^{4} f_{4} a_{0}+2 \Lambda^{2} f_{2} a_{2}+f(0) a_{4}
$$

The term in $\Lambda^{4}$ is a cosmological term, the term in $\Lambda^{2}$ is the Einstein-Hilbert action functional, and the constant term $a_{4}$ gives the integral over $M$ of curvature invariants such as the square of the Weyl curvature and topological terms such as the Gauss-Bonnet, with numerical coefficients of order one. It is thus natural to take the expression $\operatorname{Tr}(f(D / \Lambda))$ as a natural spectral formulation of gravity. We are working in the Euclidean formulation i.e. with a signature $(+,+,+,+)$ and the Euclidean space-time manifold is taken to be compact for simplicity. In the non-compact case we have shown in [10] how to replace the simple counting of eigenvalues of $|D|$ of size $<\Lambda$ given by (77), by a localized counting. This simply introduces a dilaton field. We also tested this idea of taking the expression $\operatorname{Tr}(f(D / \Lambda))$ as a natural spectral formulation of gravity by computing this expression in the case of manifolds with boundary and we found [1] that it reproduces exactly the HawkingGibbons [12 additional boundary terms which they introduced in order to restore consistency and obtain Einstein equations as the equations of motion in the case of manifolds with boundary. Further, Ashtekar et al 13, have recently shown that the use of the Dirac operator in a first order formalism, which is natural in the noncommutative setting, avoids the tuning and subtraction of a constant term. One may be worried by the large cosmological term $\Lambda^{4} f_{4} a_{4}$ that appears in the spectral action. It is large because the value of the cutoff scale $\Lambda$ is dictated, roughly speaking, by the Planck scale since the term $\Lambda^{2} f_{2} a_{2}$ is the gravitational action $\frac{1}{16 \pi G} \int R \sqrt{g} d^{4} x$. Thus it seems at first sight that the huge cosmological term $\Lambda^{4} f_{4} a_{4}$ overrides the more subtle Einstein term $\Lambda^{2} f_{2} a_{2}$. There is, however, and even at the classical level to which the present discussion applies a simple manner to overcome this difficulty. Indeed the kinematical relation (1) in fact fixes the Riemannian volume form to be5

$$
\sqrt{g} d^{4} x=\sum_{\alpha} a_{0}^{\alpha} d a_{1}^{\alpha} \wedge d a_{2}^{\alpha} \wedge d a_{3}^{\alpha} \wedge d a_{4}^{\alpha}
$$

Thus, if we vary the metric with this constraint we are in the context of unimodular gravity [14, and the cosmological term cancels out in the computation of the conditional probability of a gravitational configuration with total volume $V$ held fixed. The remaining unknown, then, is the distribution of volumes $d \mu(V)$, which is just a distribution on the half-line $\mathbb{R}_{+} \ni V$. The striking conceptual advantages of the spectral action are

\footnotetext{
${ }^{4}$ for $f$ a cutoff function

${ }^{5}$ up to a numerical factor
} 
- Simplicity: when $f$ is a cutoff function, the spectral action is just counting the number of eigenstates of $D$ in the range $[-\Lambda, \Lambda]$.

- Positivity: when $f \geq 0$ (which is the case for a cutoff function) the action $\operatorname{Tr}(f(D / \Lambda)) \geq 0$ has the correct sign for a Euclidean action.

- Invariance: one is used to the diffeomorphism invariance of the gravitational action but the functional $\operatorname{Tr}(f(D / \Lambda))$ has a much stronger invariance group, the unitary group of the Hilbert space $\mathcal{H}$.

One price to pay is that, as such, the action functional $\operatorname{Tr}(f(D / \Lambda))$ is not local. It only becomes so when it is replaced by the asymptotic expansion (8). This suggests that one should at least compute the next term in the asymptotic expansion (even though this term appears multiplied by the second derivative $f^{\prime \prime}(0)=0$ when $f$ is a cutoff function) just to get some idea of the size of the remainder. In fact both $D$ and $\Lambda$ have the physical dimension of a mass, and there is no absolute scale on which they can be measured. The ratio $D / \Lambda$ is dimensionless and the dimensionless number that governs the quality of the approximation (8) can be chosen to just be the number $N(\Lambda)$ of eigenvalues $\lambda$ of $D$ whose size is less than $\Lambda$, i.e. $|\lambda| \leq \Lambda$. When $f$ is a cutoff function the size of the error term in (8) should be $O\left(N^{-k}\right)$ for any positive $k$, using the flatness of the Taylor expansion of $f$ at 0 . In the case of interest, where $M$ is the Euclidean space-time, a rough estimate of the size of $N$ is the 4-dimensional volume of $M$ in Planck units i.e. an order of magnitude 6 of $N \sim 10^{214}$ (at the present radius, see section two for details). Thus, even without the vanishing of $f^{\prime \prime}(0)$, the rough error term $N^{-1 / 2} \sim 10^{-107}$ is quite small in the approximation of the spectral action by its local version (8). We shall in fact show that a much better estimate holds in the simplified model of Euclidean space-time given by the product $S_{a}^{3} \times S_{\beta}^{1}$. Another advantage of the above spectral description of the gravitational action is that one can now use the same action $\operatorname{Tr}(f(D / \Lambda))$ for spaces which are not Riemannian. The simplest case is the product of a Riemannian geometry $M$ (of dimension 4) by the finite space $F$ of (44). The only new term that appears is the Yang-Mills action functional of the $\mathrm{SU}(k)$ gauge fields which form the inner part of the metric. This new term appears as an additional term in the coefficient $a_{4}$ of $\Lambda^{0}$, and with the positive sign. In other words gravity on the slightly noncommutative space $M \times F$ gives ordinary gravity minimally coupled with $\mathrm{SU}(k)$-YangMills gauge theory. The latter theory is massless and the fermions are in the adjoint representation. The fermionic part of the action is easy to write since one has the operator $D$ whose inner fluctuations are

$$
D_{A}=D+A+J A J^{-1}, \quad A=\sum a_{j}\left[D, b_{j}\right], a_{j}, b_{j} \in \mathcal{A}, A=A^{*}
$$

\footnotetext{
6 using the age of the universe in Planck units to estimate the spatial Euclidean directions and the inverse temperature $\beta=1 / k T$ also in Planck units, to set the size of the imaginary time component of the Euclidean $M$.
} 
In the Einstein-Yang-Mills system so obtained, all fields involved are massless.

We now consider the product $M \times F$ of a Riemannian geometry $M$ (of dimension 4 ) by the finite space $F$ of $K O$-dimension 6 which was determined above. The computation shows that ( $c f[9]$ )

- The inner fluctuations of the metric give an $U(1) \times \mathrm{SU}(2) \times \mathrm{SU}(3)$ gauge field and a complex Higgs doublet scalar field.

- The spectral action $\operatorname{Tr}(f(D / \Lambda))$ plus the antisymmetric bilinear form $\left\langle J \xi, D_{A} \eta\right\rangle$ on chiral fermions, gives the Standard Model minimally coupled to gravity, with the Majorana mass terms and see-saw mechanism.

- The gauge couplings fulfill the unification constraint, the Yukawa couplings fulfill $Y_{2}=4 g^{2}$, where $Y_{2}$ is defined in eq(11), and the Higgs quartic coupling also fulfills a unification constraint.

Most of the new terms occur in the $a_{4}$ term of the expansion (8). This is the case for the minimal coupling of the Higgs field as well as its quartic selfinteraction. The terms $a_{0}$ and $a_{2}$ get new contributions from the Majorana masses ( $c f[9]$ ), but the main new term in $a_{2}$ has the form of a mass term for the Higgs field with the coefficient $-\Lambda^{2}$. This immediately raises the question of the meaning of the specific values of the couplings in the above action functional. Unlike the above massless Einstein-Yang-Mills system we can no longer take the above action simply as a classical action, would it be because of the unification of the three gauge couplings, which does not hold at low scale. The basic idea proposed in [8] is to consider the above action as an effective action valid at the unification scale $\Lambda$ and use the Wilsonian approach of integrating the high frequency modes to show that one obtains a realistic picture after "running down" from the unification scale to the energies at which observations are done. This approach is closely related to the approach of Reuter [15, Dou and Percacci [16, [17]. The coarse graining uses a much lower scale $\rho$ which can be understood physically as the resolution with which the system is observed. The modes with momenta larger than $\rho$ cannot be directly observed and their effect is averaged out by the functional integral. In fact the way the renormalization group is computed in [16] shows that the derivative $\rho \partial_{\rho} \Gamma_{\rho}$ of the effective action is expressed as a trace of an operator function of the propagators and is thus of a similar nature as the spectral action itself, though the trace involves all fields and not just the spin $\frac{1}{2}$ fields as in the spectral action. It is an open question to compute the renormalization group flow for the spectral action in the context of spectral triples. One expects, as explained above, that new terms involving traces of functions of the bosonic propagator 7 $\frac{\delta^{2}}{\delta D \delta D} \operatorname{Tr}(f(D / \Lambda))$ will be generated. The idea of taking the spectral action as a boundary condition of the renormalization group at unification scale

\footnotetext{
${ }^{7}$ We thank John Iliopoulos for discussions on this point.
} 
generates a number of severe tests. The first ones involve the dimensionless couplings. These include

(1) The three gauge couplings

(2) The Yukawa couplings

(3) The Higgs quartic coupling

As is well known, the gauge couplings do not unify in the Standard Model but the meeting of $g_{2}$ and $g_{3}$ specifies a "unification" scale of $\sim 10^{17} \mathrm{GeV}$. For the Yukawa couplings the boundary condition gives

$$
Y_{2}=4 g^{2}, \quad Y_{2}=\sum_{\sigma}\left(y_{\nu}^{\sigma}\right)^{2}+\left(y_{e}^{\sigma}\right)^{2}+3\left(y_{u}^{\sigma}\right)^{2}+3\left(y_{d}^{\sigma}\right)^{2} .
$$

This yields a value of the top mass which is 1.04 times the observed value when neglecting 8 the Yukawa couplings of the bottom quarks etc...and is hence compatible with experiment. The Higgs quartic coupling (scattering parameter) has the boundary condition of the form:

$$
\tilde{\lambda}(\Lambda)=g_{3}^{2} \frac{b}{a^{2}} \sim g_{3}^{2}
$$

The numerical solution to the RG equations with the boundary value $\lambda_{0}=$ 0.356 at $\Lambda=10^{17} \mathrm{GeV}$ gives $\lambda\left(M_{Z}\right) \sim 0.241$ and a Higgs mass of the order of $170 \mathrm{GeV}$. This value now seems to be ruled out experimentally but this might simply be a clear indication of the presence of some new physics, instead of the "big desert" which is assumed here in the huge range of energies between $10^{2} \mathrm{GeV}$ and $10^{17} \mathrm{GeV}$. To be more precise the above "prediction" of the Higgs mass is in perfect agreement with the one of the Standard Model, when one assumes the "big desert" ( $c f$ [19]). In a forthcoming paper [18] we show that the choice of the spectral function $f$ could play an important role, even when it varies slightly from the cutoff function. This is related to the fact that the vev of the Higgs field is proportional to the scale $\Lambda$ and thus higher order corrections do contribute. This will cause the relation between the gauge coupling constants to be modified and to change the Higgs potential. Such gravitational corrections are known to cause sizable changes to the Higgs mass [20].

The next tests involve the dimensionful couplings. These include

(1) The inverse Newton constant $Z_{g}=1 / G$.

(2) The mass term of the Higgs.

(3) The Majorana mass terms.

(4) The cosmological constant.

Since our action functional combines gravity and the Standard Model, the analysis of [16] applies, and the running of the couplings $Z$ which have the physical dimension of the square of a mass is well approximated by $\beta_{Z}=a_{1} k^{2}$ where the parameter $k$ is fixing the cutoff scale but is considered itself as one of the couplings, while the coefficient $a_{1}$ is a dimensionless

\footnotetext{
${ }^{8}$ See [9] for the precise satement.
} 
number of order one. For the inverse $Z_{g}$ of the Newton constant, one gets the solution:

$$
Z_{g}=\bar{Z}_{g}\left(1+\frac{1}{2} a_{1} \frac{k^{2}}{\bar{Z}_{g}}\right)
$$

which behaves like a constant and shows that the change in $Z_{g}$ is moderate between the low energy value $\bar{Z}_{g}$ at $k=0$ and its value at $k=m_{P}$ the Planck scale, for which $\frac{k^{2}}{Z_{g}}=1$. We have shown in [9] that a relation between the moments of the cutoff function $f$ involved in the spectral action, of the form $f_{2} \sim 5 f_{0}$ suffices to give a realistic value of the Newton constant, provided one applies the spectral action at the unification scale $\Lambda \sim 10^{17} \mathrm{GeV}$. The above discussion of the running of $Z_{g}$ shows that this yields a reasonable low energy value of the Newton constant $G$.

The form $\beta_{Z}=a_{1} k^{2}$ of the running of a coupling with mass ${ }^{2}$ dimension implies that, as a rule, even if this coupling happens to be small at low scale, it will necessarily be of the order of $\Lambda^{2}$ at unification scale. For the Majorana mass terms, we explained in 9] why they are of the order of $\Lambda^{2}$ at unification and their role in the see-saw mechanism shows that one should not expect them to be small at small scale, thus a running like (12) is realistic. Things are quite different for the mass term of the Higgs. The spectral action delivers a huge mass term of the form $-\Lambda^{2} H^{2}$ and one can check that it is consistent with the sign and order of magnitude of the quadratic divergence of the self-energy of this scalar field. However though this shows compatibility with a small low energy value it does by no means allow one to justify such a small value. Giving the term $-\Lambda^{2} H^{2}$ at unification scale and hoping to get a small value when running the theory down to low energies by applying the renormalization group, one is facing a huge fine tuning problem. Thus one should rather try to find a physical principle to explain why one obtains such a small value at low scale. In the noncommutative geometry model $M \times F$ of space-time the size of the finite space $F$ is governed by the inverse of the Higgs mass. Thus the above problem has a simple geometric interpretation: Why is the space F so larg 99 in Planck units? There is a striking similarity between this problem and the problem of the large size of space in Planck units. This suggests that it would be very worthwhile to develop cosmology in the context of the noncommutative geometry model of space-time, with in particular the preliminary step of the Lorentzian formulation of the spectral action.

This also brings us to the important role played by the dilaton field which determines the scale $\Lambda$ in the theory. The spectral action is taken to be a function of the twisted Dirac operator so that $D^{2}$ is replaced with $e^{-\phi} D^{2} e^{-\phi}$. In [10] we have shown that the spectral action is scale invariant, except for the dilaton kinetic energy. Moreover, one can show that after rescaling the

\footnotetext{
${ }^{9}$ by a factor of $10^{16}$.
} 
physical fields, the scalar potential of the theory will be independent of the dilaton at the classical level. At the quantum level, the dilaton acquires a Coleman-Weinberg potential [21] and will have a vev of the order of the Planck mass [22]. The fact that the Higgs mass is damped by a factor of $e^{-2 \phi}$, can be the basis of an explanation of the hierarchy problem.

In this paper we investigate the accuracy of the approximation of the spectral action by the first terms of its asymptotic expansion. We consider the concrete example given by the four-dimensional geometry $S_{a}^{3} \times S_{\beta}^{1}$ where $S_{a}^{3}$ is the round sphere of radius $a$ as a model of space, while $S_{\beta}^{1}$ is a circle of radius $\beta$ viewed as a model of imaginary periodic time at inverse temperature $\beta$. We compute directly the spectral action and compare it with the sum of the first terms of the asymptotic expansion. In section two we start with the round sphere $S_{a}^{3}$ and use the known spectrum of the Dirac operator together with the Poisson summation formula, to estimate the remainder when using a smooth test function. This is then applied to the four-dimensional space $S_{a}^{3} \times S_{\beta}^{1}$ where it is shown that, for natural test functions, the spectral action is completely determined by the first two terms, with an error of the order of $10^{-\sigma^{2}}$ where $\sigma$ is the inner diameter $\Lambda \mu, \mu=\inf (a, \beta)$ in units of the cutoff $\Lambda$. Thus for instance an inner diameter of 10 in cutoff units yields the accuracy of the first hundred decimal places, while an inner diameter of $10^{31}$ corresponding to the visible universe at inverse temperature of 3 Kelvin and a cutoff at Planck scale10, yields an astronomical precision of $10^{62}$ accurate decimal places. This is then extended in the presence of Higgs fields. The above direct computation allows one to double check coefficients in the spectral action. It also implies, for $S_{a}^{3} \times S_{\beta}^{1}$, the vanishing of all the Seeley-De Witt coefficients $a_{2 n}, n \geq 2$, in the heat expansion of the square of the Dirac operator. This is confirmed in section three, by a local computation of the heat kernel expansion, where it is shown that $a_{4}$ and $a_{6}$ vanish due to subtle cancelations.

\section{Estimate of the ASYmptotics}

The number $N(\Lambda)$ of eigenvalues of $|D|$ which are $\leq \Lambda$

$$
N(\Lambda)=\# \text { eigenvalues of } D \text { in }[-\Lambda, \Lambda],
$$

is a step function $N(\Lambda)$ which jumps by the integer multiplicity of an eigenvalue whenever $\Lambda$ belongs to the spectrum of $|D|$. This integer valued function is the superposition of two terms,

$$
N(\Lambda)=\langle N(\Lambda)\rangle+N_{\text {osc }}(\Lambda) .
$$

The oscillatory part $N_{\text {osc }}(\Lambda)$ is generically the same as for a random matrix. The average part $\langle N(\Lambda)\rangle$ is computed by a semiclassical approximation from

\footnotetext{
10 while the age of the universe in Planck units gives $\Lambda a \sim 10^{61}$.
} 
local expressions involving the familiar heat equation expansion and will now be carefully defined assuming an expansion of the form 11

$$
\text { Trace }\left(e^{-t \Delta}\right) \sim \sum a_{\alpha} t^{\alpha} \quad(t \rightarrow 0)
$$

for the positive operator $\Delta=D^{2}$. One has,

$$
\Delta^{-s / 2}=\frac{1}{\Gamma\left(\frac{s}{2}\right)} \int_{0}^{\infty} e^{-t \Delta} t^{s / 2-1} d t
$$

and the relation between the asymptotic expansion (14) and the $\zeta$ function,

$$
\zeta_{D}(s)=\operatorname{Trace}\left(\Delta^{-s / 2}\right)
$$

is given by,

- $\alpha<0$ gives a pole at $-2 \alpha$ for $\zeta_{D}$ with

$$
\operatorname{Res}_{s=-2 \alpha} \zeta_{D}(s)=\frac{2 a_{\alpha}}{\Gamma(-\alpha)}
$$

- $\alpha=0$ (no $\log t$ term) gives regularity at 0 for $\zeta_{D}$ with

$$
\zeta_{D}(0)=a_{0} .
$$

For simple superpositions of exponentials, as Laplace transforms,

$$
f(u)=\int_{0}^{\infty} e^{-s u} h(s) d s
$$

we can write formally,

$$
f(t \Delta)=\int_{0}^{\infty} e^{-s t \Delta} h(s) d s
$$

and

$$
\operatorname{Trace}(f(t \Delta)) \sim \sum a_{\alpha} t^{\alpha} \int_{0}^{\infty} s^{\alpha} h(s) d s .
$$

For $\alpha<0$ one has,

$$
s^{\alpha}=\frac{1}{\Gamma(-\alpha)} \int_{0}^{\infty} e^{-s v} v^{-\alpha-1} d v
$$

and

so that

$$
\int_{0}^{\infty} s^{\alpha} h(s) d s=\frac{1}{\Gamma(-\alpha)} \int_{0}^{\infty} f(v) v^{-\alpha-1} d v
$$

$$
\begin{aligned}
\operatorname{Trace}(f(t \Delta)) & \sim \sum_{\alpha<0} \frac{1}{2} \operatorname{Res}_{s=-2 \alpha} \zeta_{D}(s) \int_{0}^{\infty} f(v) v^{-\alpha-1} d v t^{\alpha} \\
& +\zeta_{D}(0) f(0)+\sum_{\alpha>0} a_{\alpha} t^{\alpha} \int_{0}^{\infty} s^{\alpha} h(s) d s .
\end{aligned}
$$

\footnotetext{
$11_{\text {the }} a_{\alpha}$ defined here is equal to the Seeley-de Witt coefficients $a_{n+2 \alpha}$ in dimension $n$.
} 
Now we assume that the only $\alpha>0$ for which $a_{\alpha} \neq 0$ are integers and note that

$$
\int_{0}^{\infty} s^{n} h(s) d s=(-1)^{n} f^{(n)}(0),
$$

so that all the terms $a_{\alpha}$ for $\alpha>0$ have vanishing coefficients when $f$ is a cutoff function which is constant equal to 1 in a neighborhood of 0 . To define the average part we consider the limit case $f(v)=1$ for $|v| \leq 1$ and 0 elsewhere and get for the coefficients of (22)

$$
\frac{1}{2} \int_{0}^{\infty} f(v) v^{-\alpha-1} d v t^{\alpha}=\frac{t^{\alpha}}{(-2 \alpha)},
$$

which, with $t=\Lambda^{-2}$, gives the following definition for the average part

$$
\langle N(\Lambda)\rangle:=\sum_{k>0} \frac{\Lambda^{k}}{k} \operatorname{Res}_{s=k} \zeta_{D}(s)+\zeta_{D}(0) .
$$

To get familiar with this definition we shall work out its meaning in a simple case,

Proposition 1. Assume that Spec $D \subset \mathbb{Z}$ and that the total multiplicity of $\{ \pm n\}$ is $P(n)$ for a polynomial $P(x)=\sum c_{k} x^{k}$. Then one has

$$
\langle N(\Lambda)\rangle=\int_{0}^{\Lambda} P(u) d u+c, \quad c=\sum c_{k} \zeta(-k),
$$

where $\zeta$ is the Riemann zeta function.

Proof. One has by construction, with $P(x)=\sum c_{k} x^{k}$,

$$
\zeta_{D}(s)=\sum P(n) n^{-s}=\sum c_{k} \zeta(s-k)
$$

Thus

$$
\operatorname{Res}_{s=k} \zeta_{D}(s)=c_{k-1}
$$

and

$$
\langle N(\Lambda)\rangle:=\sum_{k>0} \frac{\Lambda^{k}}{k} c_{k-1}+\zeta_{D}(0) .
$$

The constant $\zeta_{D}(0)$ is given by

$$
\sum c_{k} \zeta(-k)
$$

and is independent of $\Lambda$. 
2.1. The sphere $S^{4}$. We check the hypothesis of Proposition 1 for a round even sphere. We recall ([26]) that the spectrum of the Dirac operator for the round sphere $S^{n}$ of unit radius is given by

$$
\operatorname{Spec}(D)=\left\{ \pm\left(\frac{n}{2}+k\right) \mid k \in \mathbb{Z}, k \geq 0\right\}
$$

where the multiplicity of $\left(\frac{n}{2}+k\right)$ is equal to $2^{\left[\frac{n}{2}\right]}\left(\begin{array}{c}k+n-1 \\ k\end{array}\right)$. Thus for $n=4$ one gets that the spectrum consists of the relative integers, except for $\{-1,0,1\}$. The multiplicity of the eigenvalue $m$ is $4\left(\begin{array}{c}k+3 \\ k\end{array}\right)$ for $k+2=m$ which gives, for the total multiplicity of $\pm m$

$$
P(m)=\frac{4}{3}(m+1) m(m-1)=\frac{4}{3}\left(m^{3}-m\right)
$$

which shows that one gets the correct minus sign for the scalar curvature term after integration using Proposition 1. Thus one gets (up to the normalization factor $\frac{4}{3}$ )

$$
\operatorname{Tr}\left(|D|^{-s}\right)=\zeta(s-3)-\zeta(s-1)
$$

This function has a value at $s=0$ given by

$$
\zeta(-3)-\zeta(-1)=\frac{1}{120}+\frac{1}{12}=\frac{11}{120}
$$

which, taking into account the factor $\frac{4}{3}$ from normalization, matches the coefficient $\frac{11}{360} \times 4$ which appears in the spectral action in front of the GaussBonnet term, as will be shown in 93

2.2. The sphere $S^{3}$. We now want to look at the case of $S^{3}$ and determine how good the approximation of (22) is for test functions.

In order to estimate the remainder of (22) in this special case we shall use the Poisson summation formula

$$
\sum_{\mathbb{Z}} h(n)=\sum_{\mathbb{Z}} \hat{h}(n), \quad \hat{h}(x)=\int_{\mathbb{R}} h(u) e^{-2 \pi i x u} d u
$$

or rather, since the spectrum is $\frac{1}{2}+\mathbb{Z}$ in the odd case, the variant

$$
\sum_{\mathbb{Z}} g\left(n+\frac{1}{2}\right)=\sum_{\mathbb{Z}}(-1)^{n} \hat{g}(n)
$$

(obtained from (28) using $h(u)=g\left(u+\frac{1}{2}\right)$ ).

In the case of the three sphere, the eigenvalues are $\pm\left(\frac{3}{2}+k\right)$, for $k \geq 0$ with the multiplicity $2\left(\begin{array}{c}k+2 \\ k\end{array}\right)$. Thus $n+\frac{1}{2}$ has multiplicity $n(n+1)$. This holds not only for $n \geq 0$ but also for $n \in \mathbb{Z}$ since the multiplicity of $-\left(n+\frac{1}{2}\right)$ is $n(n+1)=m(m+1)$ for $m=-n-1$. In particular $\pm \frac{1}{2}$ is not in the spectrum. Thus when we evaluate $\operatorname{Tr}(f(D / \Lambda))$, with $f$ an even function, we get the following sum

$$
\operatorname{Tr}(f(D / \Lambda))=\sum_{\mathbb{Z}} n(n+1) f\left(\left(n+\frac{1}{2}\right) / \Lambda\right)
$$


We apply (29) with $g(u)=\left(u^{2}-\frac{1}{4}\right) f(u / \Lambda)$. The Fourier transform of $g$ is

$$
\begin{gathered}
\hat{g}(x)=\int_{\mathbb{R}} g(u) e^{-2 \pi i x u} d u=\int_{\mathbb{R}}\left(u^{2}-\frac{1}{4}\right) f(u / \Lambda) e^{-2 \pi i x u} d u \\
=\Lambda^{3} \int_{\mathbb{R}} v^{2} f(v) e^{-2 \pi i \Lambda x v} d v-\frac{1}{4} \Lambda \int_{\mathbb{R}} f(v) e^{-2 \pi i \Lambda x v} d v
\end{gathered}
$$

We introduce the function $\hat{f}^{(2)}$ which is the Fourier transform of $v^{2} f(v)$ and we thus get from (29),

$$
\operatorname{Tr}(f(D / \Lambda))=\Lambda^{3} \sum_{\mathbb{Z}}(-1)^{n} \hat{f}^{(2)}(\Lambda n)-\frac{1}{4} \Lambda \sum_{\mathbb{Z}}(-1)^{n} \hat{f}(\Lambda n)
$$

If we take the function $f$ in the Schwartz space $\mathcal{S}(\mathbb{R})$, then both $\hat{f}$ and $\hat{f}^{(2)}$ have rapid decay and we can estimate the sums

$$
\sum_{n \neq 0}|\hat{f}(\Lambda n)| \leq C_{k} \Lambda^{-k}, \quad \sum_{n \neq 0}\left|\hat{f}^{(2)}(\Lambda n)\right| \leq C_{k} \Lambda^{-k}
$$

which gives, for any given $k$, an estimate for a sphere of radius $a$ of the form:

$$
\operatorname{Tr}(f(D / \Lambda))=(\Lambda a)^{3} \int_{\mathbb{R}} v^{2} f(v) d v-\frac{1}{4}(\Lambda a) \int_{\mathbb{R}} f(v) d v+O\left((\Lambda a)^{-k}\right)
$$

The radius simply rescales $D$ and enters in such a way as to make the product $\Lambda a$ dimensionless. This can be seen by noting that the ratio $\frac{D}{\Lambda}$ contains the term $\frac{1}{\Lambda} e_{\alpha}^{\mu} \gamma^{\alpha} \partial_{\mu}$ and the radius enters as $\frac{1}{a}$ in the inverse dreibein $e_{\alpha}^{\mu}$. Note that, provided that $k>1$ one controls the constant in front of $(\Lambda a)^{-k}$ from the constants $c_{j}$ with

$$
\left|x^{k} \hat{f}(x)\right| \leq c_{1}, \quad\left|x^{k} \hat{f}^{(2)}(x)\right| \leq c_{2} .
$$

To get an estimate of these constants $c_{j}$, say for $k=2$, one can use the $L^{1}$-norms of the functions $\Delta f(v)$ and $\Delta\left(v^{2} f(v)\right)$ where $\Delta=-\partial_{v}^{2}$ is the Laplacian. If we take for $f$ a smooth cutoff function we thus get that the $c_{j}$ are of order one.

In fact we shall soon get a much better estimate (Corollary 4 below) which will show that, for suitable test functions, a size of $N$ in cutoff units, $\Lambda a \sim N$, already ensures a precision of the order of $e^{-N^{2}}$. We shall work directly with the physically more relevant model consisting of the product $S^{3} \times S^{1}$ viewed as a model of the imaginary time periodic compactification of space-time at a given temperature. Our estimates will work well for a size in cutoff units as small as $N \sim 10$ and will give the result with an astronomical precision for larger values. These correspond to later times since both the radius of space and the inverse temperature are increasing functions of time in this simple model. 
2.3. The product $S^{3} \times S^{1}$. We now want to move to the 4-dimensional Euclidean case obtained by taking the product $M=S^{3} \times S^{1}$ of $S^{3}$ by a small circle. We take the product geometry of a three dimensional geometry with Dirac operator $D_{3}$ by the one dimensional circle geometry with Dirac

$$
D_{1}=\frac{1}{\beta} i \nabla_{\theta}
$$

so that the spectrum of $D_{1}$ is $\frac{1}{\beta}\left(\mathbb{Z}+\frac{1}{2}\right)$.

Lemma 2. Let $D$ be the Dirac operator of the product geometry

$$
D=\left(\begin{array}{cc}
0 & D_{3} \otimes 1+i \otimes D_{1} \\
D_{3} \otimes 1-i \otimes D_{1} & 0
\end{array}\right)
$$

The asymptotic expansion for $\Lambda \rightarrow \infty$ of the spectral action of $D$ is given by

$$
\operatorname{Tr}\left(h\left(D^{2} / \Lambda^{2}\right)\right) \sim 2 \beta \Lambda \operatorname{Tr}\left(k\left(D_{3}^{2} / \Lambda^{2}\right)\right),
$$

where the function $k$ is given by

$$
k(x)=\int_{x}^{\infty}(u-x)^{-1 / 2} h(u) d u
$$

Proof. By linearity of both sides in the function $h$ (using the linearity of the transformation (36) ) it is enough to prove the result for the function $h(x)=e^{-b x}$. One has

$$
D^{2}=\left(\begin{array}{cc}
D_{3}^{2} \otimes 1+1 \otimes D_{1}^{2} & 0 \\
0 & D_{3}^{2} \otimes 1+1 \otimes D_{1}^{2}
\end{array}\right)
$$

and

$$
\operatorname{Tr}\left(e^{-b D^{2} / \Lambda^{2}}\right)=2 \operatorname{Tr}\left(e^{-b D_{1}^{2} / \Lambda^{2}}\right) \operatorname{Tr}\left(e^{-b D_{3}^{2} / \Lambda^{2}}\right)
$$

Moreover by (33) the spectrum of $D_{1}$ is $\frac{1}{\beta}\left(\mathbb{Z}+\frac{1}{2}\right)$ so that, using (29), and for fixed $\beta$ and $b$, one has for all $k>0$,

$$
\operatorname{Tr}\left(e^{-b D_{1}^{2} / \Lambda^{2}}\right) \sim \sqrt{\pi} \beta \Lambda b^{-1 / 2}+O\left(\Lambda^{-k}\right) .
$$

Thus

$$
\operatorname{Tr}\left(e^{-b D^{2} / \Lambda^{2}}\right)=2 \beta \Lambda \operatorname{Tr}\left(\sqrt{\pi} b^{-1 / 2} e^{-b D_{3}^{2} / \Lambda^{2}}\right)+O\left(\Lambda^{-k+3}\right)
$$

and the equality (35) follows from

$$
\int_{x}^{\infty}(u-x)^{-1 / 2} e^{-b u} d u=\sqrt{\pi} b^{-1 / 2} e^{-b x}
$$

which shows that the function $k$ associated to $h(x)=e^{-b x}$ by the linear transformation (36) is $k(x)=\sqrt{\pi} b^{-1 / 2} e^{-b x}$.

One can write (36) in the form

$$
k(x)=\int_{0}^{\infty} v^{-1 / 2} h(x+v) d v,
$$


which shows that $k$ has right support contained in the right support of $h$ i.e. that if $h$ vanishes identically on $[a, \infty[$ so does $k$. It also gives a good estimate of the derivatives of $k$ since

$$
\partial_{x}^{n} k(x)=\int_{0}^{\infty} v^{-1 / 2} \partial_{x}^{n} h(x+v) d v .
$$

In fact, in order to estimate the size of the remainder in the asymptotic expansion of the spectral action for the product $M=S^{3} \times S^{1}$, we shall now use the two dimensional form of (29),

$$
\sum_{\mathbb{Z}^{2}} g\left(n+\frac{1}{2}, m+\frac{1}{2}\right)=\sum_{\mathbb{Z}^{2}}(-1)^{n+m} \hat{g}(n, m)
$$

where the Fourier transform is given by

$$
\hat{g}(x, y)=\int_{\mathbb{R}^{2}} g(u, v) e^{-2 \pi i(x u+y v)} d u d v
$$

For the operator $D$ of (34), and taking for $D_{3}$ the Dirac operator of the 3 -sphere $S_{a}^{3}$ of radius $a$, the eigenvalues of $D^{2} / \Lambda^{2}$ are obtained by collecting the following

$$
\left(\frac{1}{2}+n\right)^{2}(\Lambda a)^{-2}+\left(\frac{1}{2}+m\right)^{2}(\Lambda \beta)^{-2}, \quad n, m \in \mathbb{Z}
$$

with the multiplicity $2 n(n+1)$ for each $n, m \in \mathbb{Z}$. Thus, more precisely

$$
\operatorname{Tr}\left(h\left(D^{2} / \Lambda^{2}\right)\right)=\sum_{\mathbb{Z}^{2}} 2 n(n+1) h\left(\left(\frac{1}{2}+n\right)^{2}(\Lambda a)^{-2}+\left(\frac{1}{2}+m\right)^{2}(\Lambda \beta)^{-2}\right)
$$

which is of the form:

$$
\operatorname{Tr}\left(h\left(D^{2} / \Lambda^{2}\right)\right)=\sum_{\mathbb{Z}^{2}} g\left(n+\frac{1}{2}, m+\frac{1}{2}\right)
$$

where

$$
g(u, v)=2\left(u^{2}-\frac{1}{4}\right) h\left(u^{2}(\Lambda a)^{-2}+v^{2}(\Lambda \beta)^{-2}\right)
$$

One has

$$
\begin{gathered}
\hat{g}(0,0)=\int_{\mathbb{R}^{2}} g(u, v) d u d v=2 \int_{\mathbb{R}^{2}}\left(u^{2}-\frac{1}{4}\right) h\left(u^{2}(\Lambda a)^{-2}+v^{2}(\Lambda \beta)^{-2}\right) d u d v \\
=2(\Lambda a)(\Lambda \beta) \int_{\mathbb{R}^{2}}\left((\Lambda a)^{2} x^{2}-\frac{1}{4}\right) h\left(x^{2}+y^{2}\right) d x d y
\end{gathered}
$$

using $u=x(\Lambda a)$ and $v=y(\Lambda \beta)$. Thus we get:

$$
\hat{g}(0,0)=2 \pi(\Lambda \beta)(\Lambda a)^{3} \int_{0}^{\infty} h\left(\rho^{2}\right) \rho^{3} d \rho-\pi(\Lambda \beta)(\Lambda a) \int_{0}^{\infty} h\left(\rho^{2}\right) \rho d \rho
$$

To estimate the remainder, given by the sum

$$
\sum_{(n, m) \neq(0,0)}(-1)^{n+m} \hat{g}(n, m)
$$


we treat separately the Fourier transforms of $g_{1}(u, v)=u^{2} h\left(u^{2}(\Lambda a)^{-2}+v^{2}(\beta \Lambda)^{-2}\right), \quad g_{2}(u, v)=h\left(u^{2}(\Lambda a)^{-2}+v^{2}(\Lambda \beta)^{-2}\right)$

One has

$$
\begin{gathered}
\hat{g}_{2}(n, m)=\int_{\mathbb{R}^{2}} g_{2}(u, v) e^{-2 \pi i(n u+m v)} d u d v \\
=\Lambda^{2} \beta a \int_{\mathbb{R}^{2}} h\left(x^{2}+y^{2}\right) e^{-2 \pi i(n \Lambda a x+m \Lambda \beta y)} d x d y=\Lambda^{2} \beta a \kappa_{2}(n \Lambda a, m \Lambda \beta)
\end{gathered}
$$

where the function of two variables $\kappa_{2}(u, v)$ is the Fourier transform,

$$
\kappa_{2}(u, v)=\int_{\mathbb{R}^{2}} h\left(x^{2}+y^{2}\right) e^{-2 \pi i(u x+v y)} d x d y=\kappa\left(u^{2}+v^{2}\right)
$$

The function $\kappa$ is related to the function $k(x)$ defined by (36), and one has

$$
\kappa\left(u^{2}\right)=\int_{\mathbb{R}} k\left(x^{2}\right) e^{-2 \pi i u x} d x
$$

so that $\kappa\left(u^{2}\right)$ is the Fourier transform of $k\left(x^{2}\right)$.

For $g_{1}$ one has, similarly,

$$
\begin{gathered}
\hat{g}_{1}(n, m)=\int_{\mathbb{R}^{2}} g_{1}(u, v) e^{-2 \pi i(n u+m v)} d u d v \\
=\Lambda^{4} \beta a^{3} \int_{\mathbb{R}^{2}} x^{2} h\left(x^{2}+y^{2}\right) e^{-2 \pi i(n \Lambda a x+m \Lambda \beta y)} d x d y=\Lambda^{4} \beta a^{3} \kappa_{1}(n \Lambda a, m \Lambda \beta)
\end{gathered}
$$

where the function of two variables $\kappa_{1}(u, v)$ is the Fourier transform,

$$
\kappa_{1}(u, v)=\int_{\mathbb{R}^{2}} x^{2} h\left(x^{2}+y^{2}\right) e^{-2 \pi i(u x+v y)} d x d y
$$

which is given in terms of (43) by

$$
\kappa_{1}(u, v)=-\pi^{-2}\left(u^{2} \kappa^{\prime \prime}\left(u^{2}+v^{2}\right)+\frac{1}{2} \kappa^{\prime}\left(u^{2}+v^{2}\right)\right)
$$

Now for any test function $h$ in the Schwartz space $\mathcal{S}(\mathbb{R})$, the function $x^{2} h\left(x^{2}+y^{2}\right)$ is in the Schwartz space $\mathcal{S}\left(\mathbb{R}^{2}\right)$ and thus we have for its Fourier transform, and any $k>0$, an estimate of the form

$$
\left|\kappa_{1}(u, v)\right| \leq C_{k}\left(u^{2}+v^{2}\right)^{-k}
$$

We thus get, for $k>2$,

$$
\begin{gathered}
\left|\sum_{(n, m) \neq(0,0)}(-1)^{n+m} \hat{g}_{1}(n, m)\right| \leq \sum_{(n, m) \neq(0,0)}\left|\hat{g}_{1}(n, m)\right| \\
=\Lambda^{4} \beta a^{3} \sum_{(n, m) \neq(0,0)}\left|\kappa_{1}(n \Lambda a, m \Lambda \beta)\right| \leq C_{k} \Lambda^{4} \beta a^{3} \sum_{(n, m) \neq(0,0)}\left((n \Lambda a)^{2}+(m \Lambda \beta)^{2}\right)^{-k} \\
\leq C_{k} \Lambda^{4} \beta a^{3}(\Lambda \mu)^{-2 k} \sum_{(n, m) \neq(0,0)}\left(n^{2}+m^{2}\right)^{-k}, \quad \mu=\inf (a, \beta)
\end{gathered}
$$

We thus get, using a similar estimate for $\hat{g}_{2}$, 
Theorem 3. Consider the product geometry $S_{a}^{3} \times S_{\beta}^{1}$. Then one has, for any test function $h$ in the Schwartz space $\mathcal{S}(\mathbb{R})$, the equality

$$
\operatorname{Tr}\left(h\left(D^{2} / \Lambda^{2}\right)\right)=2 \pi \Lambda^{4} \beta a^{3} \int_{0}^{\infty} h\left(\rho^{2}\right) \rho^{3} d \rho-\pi \Lambda^{2} \beta a \int_{0}^{\infty} h\left(\rho^{2}\right) \rho d \rho+\epsilon(\Lambda)
$$

where $\epsilon(\Lambda)=O\left(\Lambda^{-k}\right)$ for any $k$ is majorized by

$$
|\epsilon(\Lambda)| \leq 2 \Lambda^{4} \beta a^{3} \sum_{(n, m) \neq(0,0)}\left|\kappa_{1}(n \Lambda a, m \Lambda \beta)\right|+\frac{1}{2} \Lambda^{2} \beta a \sum_{(n, m) \neq(0,0)}\left|\kappa_{2}(n \Lambda a, m \Lambda \beta)\right| .
$$

with $\kappa_{j}$ defined in (43) and (45).

This implies that all the Seeley coefficients $a_{2 n}$ vanish for $n \geq 2$, and we shall check this directly for $a_{4}$ and $a_{6}$ in 93 .

This vanishing of the Seeley coefficients does not hold for the 4 sphere and it is worth understanding why one cannot expect to use the Poisson summation in the same way for the 4 sphere. The problem when one tries to use the Poisson formula as above is that, e.g. for the heat kernel, one is dealing with a function like $|x| e^{-t x^{2}}$ which is not smooth and whose Fourier transform does not have rapid decay at $\infty$.

2.4. Specific test functions. We shall now concretely evaluate the remainder in Theorem 3 for analytic test functions of the form

$$
h(x)=P(\pi x) e^{-\pi x}
$$

where $P$ is a polynomial of degree $d$. The Fourier transforms of the functions of two variables $x^{2} h\left(x^{2}+y^{2}\right)$ and $h\left(x^{2}+y^{2}\right)$ are of the form

$$
\kappa_{j}(u, v)=P_{j}(u, v) e^{-\pi\left(u^{2}+v^{2}\right)}
$$

where the $P_{j}$ are polynomials. More precisely, since the Fourier transform of $e^{-\lambda \pi\left(x^{2}+y^{2}\right)}$ is $\frac{1}{\lambda} e^{-\pi \frac{\left(u^{2}+v^{2}\right)}{\lambda}}$ one obtains the formula for $P_{2}$ by differentiation at $\lambda=1$ and get

$$
\kappa_{2}(u, v)=P\left(-\partial_{\lambda}\right)_{\lambda=1} \frac{1}{\lambda} e^{-\pi \frac{\left(u^{2}+v^{2}\right)}{\lambda}}
$$

which is of the form

$$
\kappa_{2}(u, v)=Q\left(\pi\left(u^{2}+v^{2}\right)\right) e^{-\pi\left(u^{2}+v^{2}\right)}
$$

where $Q$ is a polynomial of degree $d$. The transformation $P \mapsto Q=T(P)$ is given by

$$
Q(z)=P\left(-\partial_{\lambda}\right)_{\lambda=1} \frac{1}{\lambda} e^{-\frac{z}{\lambda}}
$$

Moreover one then gets

$$
\begin{gathered}
\kappa_{1}(u, v)=-(2 \pi)^{-2} \partial_{u}^{2} \kappa_{2}(u, v) \\
=\left(u^{2} Z_{1}\left(\pi\left(u^{2}+v^{2}\right)\right)+Z_{2}\left(\pi\left(u^{2}+v^{2}\right)\right)\right) e^{-\pi\left(u^{2}+v^{2}\right)}
\end{gathered}
$$


where

$$
Z_{1}=-Q+2 Q^{\prime}-Q^{\prime \prime}, \quad Z_{2}=\frac{1}{2 \pi}\left(Q-Q^{\prime}\right)
$$

We let $C_{P}$ be the sum of the absolute values of the coefficients of $Q=T(P)$.

Corollary 4. Consider the product geometry $S_{a}^{3} \times S_{\beta}^{1}$. Let $\mu=\inf (a, \beta)$. Then one has, with $h$ any test function of the form (48), the equality

$$
\operatorname{Tr}\left(h\left(D^{2} / \Lambda^{2}\right)\right)=2 \pi \Lambda^{4} \beta a^{3} \int_{0}^{\infty} h\left(\rho^{2}\right) \rho^{3} d \rho-\pi \Lambda^{2} \beta a \int_{0}^{\infty} h\left(\rho^{2}\right) \rho d \rho+\epsilon(\Lambda)
$$

where, assuming $\mu \Lambda \geq \sqrt{d(1+\log d)}$ and $\mu \Lambda \geq 1$,

$$
|\epsilon(\Lambda)| \leq C e^{-\frac{\pi}{2}(\mu \Lambda)^{2}}, C=\Lambda^{4} \beta a^{3} C_{P}\left(8+6 d+2 d^{2}\right)
$$

Proof. One has

$$
x^{k} e^{-x / 2} \leq 1, \forall x \geq 3 k(1+\log k)
$$

Thus, for $(n, m) \neq(0,0)$ one has

$$
\left|\kappa_{2}(n \Lambda a, m \Lambda \beta)\right| \leq C_{P} e^{-\frac{\pi}{2}\left((n \Lambda a)^{2}+(m \Lambda \beta)^{2}\right)}
$$

since $\pi\left((n \Lambda a)^{2}+(m \Lambda \beta)^{2}\right) \geq 3 d(1+\log d)$. Moreover, since $e^{-\frac{\pi}{2}(\mu \Lambda)^{2}} \leq \frac{1}{4}$, one gets

and

$$
\sum_{(n, m) \neq(0,0)} e^{-\frac{\pi}{2}\left((n \Lambda a)^{2}+(m \Lambda \beta)^{2}\right)} \leq 8 e^{-\frac{\pi}{2}(\mu \Lambda)^{2}}
$$

$$
\sum_{(n, m) \neq(0,0)}\left|\kappa_{2}(n \Lambda a, m \Lambda \beta)\right| \leq 8 C_{P} e^{-\frac{\pi}{2}(\mu \Lambda)^{2}}
$$

A similar estimate using (50) yields

$$
\sum_{(n, m) \neq(0,0)}\left|\kappa_{1}(n \Lambda a, m \Lambda \beta)\right| \leq\left(2+3 d+d^{2}\right) C_{P} e^{-\frac{\pi}{2}(\mu \Lambda)^{2}}
$$

Thus by Theorem 3, the inequality (52) holds for

$$
C=C_{P}\left(2 \Lambda^{4} \beta a^{3}\left(2+3 d+d^{2}\right)+4 \Lambda^{2} \beta a\right) .
$$

One then uses the hypothesis $\mu \Lambda \geq 1$ to simplify $C$.

The meaning of Corollary 4 is that the accuracy of the asymptotic expansion is at least of the order of $e^{-\frac{\pi}{2}(\mu \Lambda)^{2}}$. Indeed the term $\Lambda^{4} \beta a^{3}$ in (52) is the dominant volume term in the spectral action and the other terms in the formula for $C$ are of order one. Thus for instance for a size $\mu \Lambda \sim 100$ one gets that the asymptotic expansion accurately delivers the first 6820 decimal places of the spectral action. Note that some test functions of the form (48) give excellent approximations to cutoff functions, in particular

$$
h_{n}(x)=\sum_{0}^{n} \frac{(\pi x)^{k}}{k !} e^{-\pi x}
$$


The graph of $h_{n}\left(x^{2}\right)$ is shown in Figure 1 for $n=20$. For $h=h_{20}$ the computation gives $C_{P}\left(8+6 d+2 d^{2}\right) \leq 2 \times 10^{6}$ so that this constant only interferes with the last six decimal places in the above accuracy.

In our simplified physical model we test the approximation of the spectral action by its asymptotic expansion for the Euclidean model

$$
E(t)=S_{a(t)}^{3} \times S_{\beta(t)}^{1}
$$

where space at a given time $t$ is given by a sphere with radius $a(t)$ and $\beta(t)$ is a uniform value of inverse temperature. One can then easily see that the above approximation to the spectral action is fantastically accurate, going backwards in time all the way up to one order lower than the Planck energy. In doing so the radius $a(t)$ varies between at least $\sim 10^{61}$ Planck units and 10 Planck units (i.e. $10^{-34} \mathrm{~m}$ ), while the temperature varies between $2.7^{\circ} \mathrm{K}$ and $\left(10^{31}\right)^{\circ} \mathrm{K}$. It is for an inner size less than 10 in Planck units that the approximation does break down.

Remark 5. For later purpose it is important to estimate the constant $C_{P}$ in terms of the coefficients of the polynomial $P$. Let then $P(z)=z^{n}$. One has $h(x)=(\pi x)^{n} e^{-\pi x}$ and the function $k(x)$ associated to $h$ by (36) is

$$
\begin{gathered}
k(x)=\int_{\mathbb{R}} h\left(x+y^{2}\right) d y=\pi^{n} e^{-\pi x} \sum_{0}^{n}\left(\begin{array}{l}
n \\
k
\end{array}\right) x^{n-k} \int_{\mathbb{R}} y^{2 k} e^{-\pi y^{2}} d y \\
=\pi^{-1 / 2} e^{-\pi x} \sum_{0}^{n}\left(\begin{array}{l}
n \\
k
\end{array}\right) \Gamma\left(\frac{1}{2}+k\right)(\pi x)^{n-k}
\end{gathered}
$$

To obtain $Q=T(P)$ one then needs to compute the Fourier transform $\kappa\left(u^{2}\right)$ of the function $k\left(x^{2}\right)$ as in (44). The Fourier transform of $\left(\pi x^{2}\right)^{m} e^{-\pi x^{2}}$ is

$$
\ell_{m}(u)=(-4 \pi)^{-m} \partial_{u}^{2 m} e^{-\pi u^{2}}=L_{m}\left(\pi u^{2}\right) e^{-\pi u^{2}}
$$

and one checks, using the relation

$$
L_{m+1}(z)=1 / 2\left((1-2 z) L_{m}(z)+(-1+4 z) L_{m}^{\prime}(z)-2 z L_{m}^{\prime \prime}(z)\right.
$$

that the sign of the coefficient of $z^{k}$ in $L_{m}(z)$ is $(-1)^{k}$. Thus the sum of the absolute values of the coefficients of $L_{m}$ is equal to $L_{m}(-1)=\ell_{m}\left(i \pi^{-1 / 2}\right) / e$. Thus since the above sum giving $k(x)$ has positive coefficients we get that, for $P(z)=z^{n}$, the constant $C_{P}$ is given by $Q\left(\pi\left(u^{2}+v^{2}\right) e^{-\pi\left(u^{2}+v^{2}\right)}\right) / e$ for $(u, v)=\left(i \pi^{-1 / 2}, 0\right)$, which gives

$$
C_{P}=\int_{\mathbb{R}^{2}} \pi^{n}\left(y^{2}+x^{2}\right)^{n} e^{-\pi y^{2}-\pi x^{2}+2 \sqrt{\pi} x-1} d x d y .
$$

One then gets

$$
C_{P} \leq 2 \int_{0}^{\infty} u^{2 n+1} e^{-(u-1)^{2}} d u=O\left(\lambda^{n} n !\right), \forall \lambda>1 .
$$


Thus, for an arbitrary polynomial $P(z)=\sum_{0}^{d} a_{k} z^{k}$ one has

$$
C_{P} \leq 2 \int_{0}^{\infty}|P|\left(u^{2}\right) e^{-(u-1)^{2}} u d u, \quad|P|(z)=\sum\left|a_{k}\right| z^{k}
$$

2.5. The Higgs potential. We now look at what happens if one performs the following replacement on the operator

$$
D^{2} \mapsto D^{2}+H^{2}
$$

where $H$ is a constant. This amounts with the above notations to the replacement

$$
h(u) \mapsto h\left(u+H^{2} / \Lambda^{2}\right) .
$$

As long as $H^{2} / \Lambda^{2}$ is of order one, we can trust the asymptotic expansion and we just need to understand the effect of this shift on the two terms of (47). We look at the first contribution, i.e.

$$
2 \pi \Lambda^{4} \beta a^{3} \int_{0}^{\infty} h\left(\rho^{2}\right) \rho^{3} d \rho=\pi \Lambda^{4} \beta a^{3} \int_{0}^{\infty} u h(u) d u
$$

We let $x=H^{2} / \Lambda^{2}$, and get, after the above replacement,

$$
\begin{gathered}
\int_{0}^{\infty} u h(u+x) d u=\int_{x}^{\infty}(v-x) h(v) d v=\int_{0}^{\infty}(v-x) h(v) d v-\int_{0}^{x}(v-x) h(v) d v \\
=\int_{0}^{\infty} v h(v) d v-x \int_{0}^{\infty} h(v) d v-\int_{0}^{x}(v-x) h(v) d v
\end{gathered}
$$

The first term corresponds to the initial contribution of $\pi \Lambda^{4} \beta a^{3} \int_{0}^{\infty} u h(u) d u$. The second term gives

$$
-\pi \Lambda^{4} \beta a^{3} x \int_{0}^{\infty} h(v) d v=-\pi \Lambda^{2} \beta a^{3} H^{2} \int_{0}^{\infty} h(v) d v
$$

which is the expected Higgs mass term from the Seeley-de Witt coefficient $a_{2}$. To understand the last term we assume that $h$ is a cutoff function.

Lemma 6. If $h$ is a smooth function constant on the interval $[0, c]$, then for $x=H^{2} / \Lambda^{2} \leq c$ the new terms arising from the replacement (56) are given by

$$
-\pi \Lambda^{2} \beta a^{3} \int_{0}^{\infty} h(v) d v H^{2}+\frac{1}{2} \pi \beta a h(0) H^{2}+\frac{1}{2} \pi \beta a^{3} h(0) H^{4}
$$

Proof. For the perturbation of $\pi \Lambda^{4} \beta a^{3} \int_{0}^{\infty} u h(u) d u$, besides (57), we just need to compute the last term $-\int_{0}^{x}(v-x) h(v) d v$, and one has

$$
-\int_{0}^{x}(v-x) h(v) d v=h(0) \int_{0}^{x}(x-v) d v=\frac{1}{2} h(0) x^{2}
$$

since $h$ is constant on the interval $[0, x]$. 
We then look at the effect on the second contribution, i.e.

$$
-\pi \Lambda^{2} \beta a \int_{0}^{\infty} h\left(\rho^{2}\right) \rho d \rho=-\frac{1}{2} \pi \Lambda^{2} \beta a \int_{0}^{\infty} h(u) d u
$$

We let, as above, $x=H^{2} / \Lambda^{2}$, and get

$$
\int_{0}^{\infty} h(u+x) d u=\int_{x}^{\infty} h(v) d v=\int_{0}^{\infty} h(v) d v-\int_{0}^{x} h(v) d v
$$

Thus the perturbation, under the hypothesis of Lemma 6 is

$$
-\frac{1}{2} \pi \Lambda^{2} \beta a(-x h(0))=\frac{1}{2} \pi \beta a h(0) H^{2}
$$

The three terms in formula (58) correspond to the following new terms for the spectral action

- The Higgs mass term coming from the Seeley-de Witt coefficient $a_{2}$.

- The $R H^{2}$ term coming from the Seeley-de Witt coefficient $a_{4}$.

- The Higgs potential term in $H^{4}$ coming from the Seeley-de Witt coefficient $a_{4}$.

We can now state the analogue of Theorem 3 as follows

Theorem 7. Consider the product geometry $S_{a}^{3} \times S_{\beta}^{1}$. Let $\mu=\inf (a, \beta)$. Then one has, with $h$ any test function of the form (48), the equality

$$
\begin{gathered}
\operatorname{Tr}\left(h\left(\left(D^{2}+H^{2}\right) / \Lambda^{2}\right)\right)=2 \pi \Lambda^{4} \beta a^{3} \int_{0}^{\infty} h\left(\rho^{2}\right) \rho^{3} d \rho-\pi \Lambda^{2} \beta a \int_{0}^{\infty} h\left(\rho^{2}\right) \rho d \rho \\
+\pi \Lambda^{4} \beta a^{3} V\left(H^{2} / \Lambda^{2}\right)+\frac{1}{2} \pi \Lambda^{2} \beta a W\left(H^{2} / \Lambda^{2}\right)+\epsilon(\Lambda)
\end{gathered}
$$

where

$$
V(x)=\int_{0}^{\infty} u(h(u+x)-h(u)) d u, W(x)=\int_{0}^{x} h(u) d u
$$

and, assuming $\mu \Lambda \geq \sqrt{d(1+\log d)}, \mu \Lambda \geq 1$, and $H^{2} \Lambda^{-2} \leq c / \pi$,

$$
|\epsilon(\Lambda)| \leq C e^{-\frac{\pi}{2}(\mu \Lambda)^{2}}, C=\Lambda^{4} \beta a^{3} C_{P}^{\prime}\left(8+6 d+2 d^{2}\right)
$$

where, with $P(z)=\sum_{0}^{d} a_{k} z^{k}$ one has

$$
C_{P}^{\prime}=4 \int_{0}^{\infty}|P|\left(u^{2}+c\right) e^{-(u-1)^{2}} u d u, \quad|P|(z)=\sum\left|a_{k}\right| z^{k}
$$

Proof. The new terms simply express the replacement (56) in the formula of Theorem 3. The new function $\tilde{h}$ thus obtained is still of the form (48) since it is obtained from $h$ by a translation. It thus only remains to estimate $C_{\tilde{P}}$ where $\tilde{P}$ is the polynomial such that $\tilde{h}(u)=\tilde{P}(\pi u) e^{-\pi u}$. For $P(z)=z^{n}$ the constant $C_{\tilde{P}}$ for a translation $u \mapsto u+x, x \geq 0$ of the variable, is less than the constant $C_{P_{x}}$ for the polynomial

$$
P_{x}(\pi u)=P(\pi(u+x))=\sum\left(\begin{array}{l}
n \\
k
\end{array}\right)(\pi x)^{n-k}(\pi u)^{k}
$$


Thus, by Remark 5. (55), the constant $C_{P_{x}}$ is estimated by

$$
C_{P_{x}} \leq 2 \int_{0}^{\infty}\left(u^{2}+\pi x\right)^{n} e^{-(u-1)^{2}} u d u
$$

which is an increasing function of $x$ and thus only needs to be controlled for $x=c / \pi$ in our case.

For instance, for $h=h_{20}$ the computation gives $C_{P}^{\prime}\left(8+6 d+2 d^{2}\right) \leq 3 \times 10^{7}$ for $c=1$, so that this constant only interferes with the last seven decimal places in the accuracy which is the same as in Corollary 4 .

Moreover as shown in Lemma 6, when $h$ is close to a true cutoff function

$$
\begin{gathered}
\pi \Lambda^{4} \beta a^{3} V\left(H^{2} / \Lambda^{2}\right)+\frac{1}{2} \pi \Lambda^{2} \beta a W\left(H^{2} / \Lambda^{2}\right) \\
=-2 \pi \Lambda^{2} \beta a^{3} \int_{0}^{\infty} h\left(\rho^{2}\right) \rho d \rho H^{2}+\frac{1}{2} \pi \beta a h(0) H^{2}+\frac{1}{2} \pi \beta a^{3} h(0) H^{4}+\delta
\end{gathered}
$$

where the remainder $\delta$ is estimated from the Taylor expansion of $h$ at 0 . For instance for the functions $h_{n}$ of (53), one has by construction $0 \leq h_{n}(x) \leq 1$ for all $x$ and since

$$
h_{n}(x)=1-\sum a(n, k) x^{n+k+1}, \quad a(n, k)=(-1)^{k} /((n+k+1) n ! k !)
$$

one gets, for $h=h_{n}$ the estimate

$$
|\delta| \leq \pi \Lambda^{4} \beta a^{3} \frac{x^{n+3}}{(n+3)(n+1) !}+\pi \Lambda^{2} \beta a \frac{x^{n+2}}{2(n+2) !}, \quad x=H^{2} / \Lambda^{2} .
$$

While the function $W$ is by construction the primitive of $h$, and is increasing for $h \geq 0$ one has, under the hypothesis of positivity of $h$,

Lemma 8. The function $V(x)$ is decreasing with derivative given by

$$
V^{\prime}(x)=-\int_{x}^{\infty} h(v) d v
$$

The second derivative of $V(x)$ is equal to $h(x)$.

Proof. One has

$$
V^{\prime}(x)=\int_{0}^{\infty} u h^{\prime}(u+x) d u=[u h(u+x)]_{0}^{\infty}-\int_{0}^{\infty} h(u+x) d u
$$

which gives the required results.

\section{Seeley-De Witt coefficients and Spectral Action on $S^{3} \times S^{1}$}

In this section we shall compute the asymptotic expansion of the spectral action on the background geometry of $S^{3} \times S^{1}$ using heat kernel methods. This will enable us to check independently the accuracy of the estimates derived in the last section. This background is physically relevant since it can be connected with simple cosmological models. We refer to [23], [8] for the formulas and the method of the computation. The general method we 
use is also explained in great detail in a forthcoming paper [18]. We start by computing $a_{0}$ :

$$
\begin{aligned}
a_{0} & =\frac{\operatorname{Tr}(1)}{16 \pi^{2}} \int \sqrt{g} d^{4} x=\frac{1}{4 \pi^{2}} \int_{S^{3}} \sqrt{{ }^{3} g} d^{3} x \int_{S^{1}} d x \\
& =\frac{1}{4 \pi^{2}}\left(2 \pi^{2} a^{3}\right)(2 \pi \beta)=\pi \beta a^{3}
\end{aligned}
$$

where $\beta$ is the radius of $S_{\beta}^{1}$ and the volume of the three sphere $S_{a}^{3}$ of radius $a$ is $2 \pi^{2} a^{3}$ [25].

Next we calculate $a_{2}$

$$
a_{2}=\frac{1}{16 \pi^{2}} \int d^{4} x \sqrt{g} \operatorname{Tr}\left(E+\frac{1}{6} R\right)
$$

where $E$ is defined from the relation

$$
D^{2}=-\left(g^{\mu \nu} \nabla_{\mu} \nabla_{\nu}+E\right)
$$

where for pure gravity we have

$$
E=-\frac{1}{4} R
$$

so that (using $\operatorname{Tr}(1)=4$ )

$$
a_{2}=\frac{1}{4 \pi^{2}}\left(-\frac{R}{12}\right) \int d^{4} x \sqrt{g}
$$

since the curvature is constant. The curvature tensor 12 is, using the coordinates of [25] for the three sphere $S_{a}^{3}$ with labels $i, j, k, l$ and the label 4 for the coordinate in $S_{\beta}^{1}$,

$$
\begin{aligned}
R_{i j k l} & =-a^{-2}\left(g_{i k} g_{j l}-g_{i l} g_{j k}\right), \quad i, j, k, l=1, \cdots 3 \\
R_{i j k 4} & =0 \\
R_{i 4 j 4} & =0
\end{aligned}
$$

where $g_{i j}$ is the metric on the three sphere as in 25]. The Ricci tensor is given, following the sign convention of [24] which introduces a minus sign in passing from the curvature tensor to the Ricci tensor, by

$$
\begin{aligned}
& R_{i j}=-g^{k l} R_{i k j l}=2 a^{-2} g_{i j} \\
& R_{i 4}=0 \\
& R_{44}=0
\end{aligned}
$$

Thus the scalar curvature is

$$
R=g^{i j} R_{i j}=\frac{6}{a^{2}}
$$

\footnotetext{
12 the sign convention for this tensor is the same as in 23
} 
and the $a_{2}$ term in the heat expansion simplifies to

$$
a_{2}=-\pi \beta a\left(\frac{1}{2}\right)
$$

Next for $a_{4}$ we have

$$
\begin{aligned}
a_{4}= & \frac{1}{16 \pi^{2}} \frac{1}{360} \int_{M} d^{4} x \sqrt{g} \operatorname{Tr}\left(12 R_{; \mu}^{\mu}+5 R^{2}-2 R_{\mu \nu} R^{\mu \nu}\right. \\
& \left.+2 R_{\mu \nu \rho \sigma} R^{\mu \nu \rho \sigma}+60 R E+180 E^{2}+60 E_{; \mu}{ }^{\mu}+30 \Omega_{\mu \nu} \Omega^{\mu \nu}\right)
\end{aligned}
$$

where for the pure gravitational theory, we have

$$
E=-\frac{1}{4} R, \quad \Omega_{\mu \nu}=\frac{1}{4} R_{\mu \nu}^{a b} \gamma_{a b}
$$

In this case it was shown in [8] that $a_{4}$ reduces to

$$
\begin{aligned}
a_{4} & =\frac{1}{4 \pi^{2}} \frac{1}{360} \int d^{4} x \sqrt{g}\left(5 R^{2}-8 R_{\mu \nu}^{2}-7 R_{\mu \nu \rho \sigma}^{2}\right) \\
& =\frac{1}{4 \pi^{2}} \frac{1}{360} \int d^{4} x \sqrt{g}\left(-18 C_{\mu \nu \rho \sigma}^{2}+11 R^{*} R^{*}\right)
\end{aligned}
$$

which is obviously scale invariant. The Weyl tensor $C_{\mu \nu \rho \sigma}$ is defined by

$$
\begin{aligned}
C_{\mu \nu \rho \sigma} & =R_{\mu \nu \rho \sigma}+\frac{1}{2}\left(R_{\mu \rho} g_{\nu \sigma}-R_{\nu \rho} g_{\mu \sigma}-R_{\mu \sigma} g_{\nu \rho}+R_{\nu \sigma} g_{\mu \rho}\right) \\
& -\frac{1}{6}\left(g_{\mu \rho} g_{\nu \sigma}-g_{\nu \rho} g_{\mu \sigma}\right) R
\end{aligned}
$$

This tensor vanishes on $S^{3} \times S^{1}$ as can be seen by evaluating the components

$$
\begin{aligned}
C_{i j k l} & =a^{-2}\left[-\left(g_{i k} g_{j l}-g_{i l} g_{j k}\right)+2\left(g_{i k} g_{j l}-g_{i l} g_{j k}\right)-\left(g_{i k} g_{j l}-g_{i l} g_{j k}\right)\right]=0 \\
C_{i j k 4} & =0 \\
C_{i 4 k 4} & =0
\end{aligned}
$$

Similarly the Gauss-Bonnet term

$$
\begin{aligned}
R^{*} R^{*} & =\frac{1}{4} \epsilon^{\mu \nu \rho \sigma} \epsilon_{\alpha \beta \gamma \delta} R_{\mu \nu}{ }^{\alpha \beta} R_{\rho \sigma}{ }^{\gamma \delta} \\
& =\epsilon^{i j k 4} \epsilon_{\alpha \beta \gamma \delta}\left(R_{i j}{ }^{\alpha \beta} R_{k 4}{ }^{\gamma \delta}\right) \\
& =0
\end{aligned}
$$

The next step of calculating $a_{6}$ is in general extremely complicated, but for spaces of constant curvature the expression simplifies as all covariant derivatives of the curvature tensor, Riemann tensor and scalar curvature vanish. The non-vanishing terms are, using Theorem 4.8.16 of [23] and the 
above sign convention for the Ricci tensor $R_{\mu \nu}$ and the scalar curvature,

$$
\begin{aligned}
a_{6}=\frac{1}{16 \pi^{2}} \int d^{4} x \sqrt{g} \operatorname{Tr} & \left(\frac { 1 } { 9 \cdot 7 ! } \left(35 R^{3}-42 R R_{\mu \nu}^{2}+42 R R_{\mu \nu \rho \sigma}^{2}\right.\right. \\
- & 208 R_{\mu \nu} R_{\mu \rho} R_{\nu \rho}-192 R_{\mu \rho} R_{\nu \sigma} R_{\mu \nu \rho \sigma}-48 R_{\mu \nu} R_{\mu \rho \sigma \kappa} R_{\nu \rho \sigma \kappa} \\
- & \left.44 R_{\mu \nu \rho \sigma} R_{\mu \nu \kappa \lambda} R_{\rho \sigma \kappa \lambda}-80 R_{\mu \nu \rho \sigma} R_{\mu \kappa \rho \lambda} R_{\nu \kappa \sigma \lambda}\right) \\
+\frac{1}{360}(- & 12 \Omega_{\mu \nu} \Omega_{\nu \rho} \Omega_{\rho \mu}-6 R_{\mu \nu \rho \sigma} \Omega_{\mu \nu} \Omega_{\rho \sigma}-4 R_{\mu \nu} \Omega_{\mu \rho} \Omega_{\nu \rho}+5 R \Omega_{\mu \nu}^{2} \\
& \left.\left.+60 E^{3}+30 E \Omega_{\mu \nu}^{2}+30 R E^{2}+5 R^{2} E-2 R_{\mu \nu}^{2} E+2 R_{\mu \nu \rho \sigma}^{2} E\right)\right)
\end{aligned}
$$

We can now compute each of the above eighteen terms. These are listed in an appendix. Collecting these terms we obtain that the integrand is

$$
\begin{aligned}
& -\frac{4 a^{-6}}{9 \cdot 7 !}\left(-35 \cdot 6^{3}+42 \cdot 72-42 \cdot 72+208 \cdot 24-192 \cdot 24+48 \cdot 24-44 \cdot 24-80 \cdot 6\right) \\
& -\frac{4 a^{-6}}{360}\left(9+18-12+45+\frac{15 \cdot 27}{2}-\frac{5 \cdot 27}{2}-15 \cdot 27+10 \cdot 27-36+36\right) \\
& =a^{-6}\left(\frac{2}{3}-\frac{2}{3}\right)=0
\end{aligned}
$$

implying that

$$
a_{6}=0,
$$

which shows that the cancelation is highly non-trivial. We conclude that the spectral action, up to terms of order $\frac{1}{\Lambda^{4}}$ is given by

$$
S=\Lambda^{4} \int_{0}^{\infty} x h(x) d x\left(\pi \beta a^{3}\right)-\Lambda^{2} \int_{0}^{\infty} h(x) d x\left(\pi \beta a \frac{1}{2}\right)+O\left(\Lambda^{-4}\right)
$$

After making the change of variables $x=\rho^{2}$ we get

$$
S=(\pi \beta \Lambda)\left[2(\Lambda a)^{3} \int_{0}^{\infty} \rho^{3} h\left(\rho^{2}\right) d \rho-(\Lambda a) \int_{0}^{\infty} \rho h\left(\rho^{2}\right) d \rho\right]+O\left(\Lambda^{-4}\right)
$$

This confirms equation (47) and shows that, to a very high degree of accuracy, the spectral action on $S^{3} \times S^{1}$ is given by the first two terms.

Remark 9. It is worth noting that one can also check the value of the Gauss-Bonnet term on $S^{4}$ and show that it agrees with the value obtained in (27). To see this note that the Riemann tensor in this case is given by $([25])$

$$
R_{\mu \nu \rho \sigma}=-a^{-2}\left(g_{\mu \rho} g_{\nu \sigma}-g_{\mu \sigma} g_{\nu \rho}\right)
$$

which implies 13 that

$$
\begin{aligned}
C_{\mu \nu \rho \sigma} & =0 \\
R^{*} R^{*} & =6 a^{-4}
\end{aligned}
$$

\footnotetext{
${ }^{13}$ One can double check the value of $R^{*} R^{*}$ using the Gauss-Bonnet Theorem.
} 
and thus

$$
a_{4}=\frac{1}{4 \pi^{2}} \frac{11}{60} a^{-4} \int_{S^{4}} d^{4} x \sqrt{g}
$$

The volume of $S^{4}$ is

$$
V_{4}=\int_{S^{4}} d^{4} x \sqrt{g}=\frac{2 \pi^{\frac{5}{2}}}{\Gamma\left(\frac{5}{2}\right)} a^{4}=\frac{8 \pi^{2}}{3} a^{4}
$$

and this implies that

$$
a_{4}=\frac{11}{360} \times 4
$$

which agrees exactly with the calculation of (27) based on zeta functions.

\section{Appendix}

In this appendix we compute the eighteen non-vanishing terms that appear in the $a_{6}$ term of the heat kernel expansion. Using the properties

$$
\begin{aligned}
& R_{\mu \nu}^{2}=R_{i j}^{2}=12 a^{-4} \\
& R_{\mu \nu \rho \sigma}^{2}=R_{i j k l}^{2}=12 a^{-4} \\
35 R^{3}= & 35(6)^{3} a^{-6} \\
-42 R R_{\mu \nu}^{2}= & -42(6)(12) a^{-6} \\
-208 R_{\mu \nu} R_{\mu \rho} R_{\nu \rho}= & -208(2)^{3} g_{i j} g_{i k} g_{j k}=208(2)^{3}(3) a^{-6} \\
-192 R_{\mu \rho} R_{\nu \sigma} R_{\mu \nu \rho \sigma}= & -192 R_{i k} R_{j l} R_{i j k l} \\
= & 192(2)^{2} g_{i k} g_{j l}\left(g_{i k} g_{j l}-g_{i l} g_{j k}\right) a^{-6} \\
= & 192(24) a^{-6} \\
-48 R_{\mu \nu} R_{\mu \rho \sigma \kappa} R_{\nu \rho \sigma \kappa}= & -48(2) g_{i j}\left(g_{i k} g_{l m}-g_{i l} g_{k m}\right)\left(g_{j k} g_{l m}-g_{j l} g_{k m}\right) a^{-6} \\
= & -48(4) g_{i j}\left(2 g_{i j}\right) a^{-6}=-48(24) a^{-6} \\
-44 R_{\mu \nu \rho \sigma} R_{\mu \nu \kappa \lambda} R_{\rho \sigma \kappa \lambda}= & 44\left(g_{i k} g_{j l}-g_{i l} g_{j k}\right)\left(g_{i p} g_{j q}-g_{i q} g_{j p}\right)\left(g_{k p} g_{l q}-g_{l q} g_{l p}\right) a^{-6} \\
= & 44(4)(6) a^{-6} \\
-80 R_{\mu \nu \rho \sigma} R_{\mu \kappa \rho \lambda} R_{\nu \kappa \sigma \lambda}= & 80\left(g_{i k} g_{j l}-g_{i l} g_{j k}\right)\left(g_{i k} g_{p q}-g_{i q} g_{p k}\right)\left(g_{j l} g_{p q}-g_{j q} g_{p l}\right) a^{-6} \\
= & 80\left(3 g_{j l} g_{p q}-g_{p q} g_{j l}-g_{l j} g_{p q}+g_{l q} g_{j p}\right)\left(g_{j l} g_{p q}-g_{j q} g_{p l}\right) a^{-6} \\
= & 80(9-3+3-3) a^{-6} \\
= & 80(6) a^{-6}
\end{aligned}
$$

Collecting the first set of terms we get

$$
\begin{aligned}
& -\frac{4 a^{-6}}{9 \cdot 7 !}\left(-35 \cdot 6^{3}+42 \cdot 72-42 \cdot 72+208 \cdot 24-192 \cdot 24+48 \cdot 24-44 \cdot 24-80 \cdot 6\right) \\
& =\frac{2}{3} a^{-6}
\end{aligned}
$$


Now we continue with the second set of terms

$$
\begin{aligned}
-12 \operatorname{Tr}\left(\Omega_{\mu \nu} \Omega_{\nu \rho} \Omega_{\rho \mu}\right) & =-12\left(\frac{1}{4}\right)^{3} \operatorname{Tr}\left(\gamma_{a b} \gamma_{c d} \gamma_{e f}\right) R_{\mu \nu}{ }^{a b} R_{\nu \rho}{ }^{c d} R_{\rho \mu}{ }^{e f} \\
& =\operatorname{Tr}(1) 12\left(\frac{1}{4}\right)^{3}(8) R_{\mu \nu a b} R_{\nu \rho b c} R_{\rho \mu a c} \\
& =-\frac{3}{2}\left(g_{i k} g_{j l}-g_{i l} g_{j k}\right)\left(g_{j l} g_{p q}-g_{j p} g_{l q}\right)\left(g_{p k} g_{i q}-g_{p i} g_{k q}\right) a^{-6} \operatorname{Tr}(1) \\
& =-\frac{3}{2}\left(3 g_{i k} g_{p q}-g_{i k} g_{p q}-g_{i k} g_{p q}+g_{i q} g_{p k}\right)\left(g_{p k} g_{i q}-g_{p i} g_{k q}\right) 4 a^{-6} \\
& =-\frac{3}{2}(3-3+9-3) 4 a^{-6} \\
& =-9 \cdot 4 a^{-6}
\end{aligned}
$$

$$
\begin{aligned}
-6 R_{\mu \nu \rho \sigma} \operatorname{Tr}\left(\Omega_{\mu \nu} \Omega_{\rho \sigma}\right) & =-\frac{6}{4^{2}} R_{\mu \nu \rho \sigma} \operatorname{Tr}\left(\gamma_{a b} \gamma_{c d}\right) R_{\mu \nu}^{a b} R_{\rho \sigma}{ }^{c d} \\
& =\frac{12}{16} R_{\mu \nu \rho \sigma} R_{\mu \nu}^{a b} R_{\rho \sigma a b} \operatorname{Tr}(1) \\
& =-\frac{3}{4}\left(g_{i k} g_{j l}-g_{i l} g_{j k}\right)\left(g_{i p} g_{j q}-g_{i q} g_{j p}\right)\left(g_{k p} g_{l q}-g_{k q} g_{l p}\right) 4 a^{-6} \\
& =-3(9-3) 4 a^{-6}=-18 \cdot 4 a^{-6}
\end{aligned}
$$

$$
\begin{aligned}
-4 R_{\mu \nu} \operatorname{Tr}\left(\Omega_{\mu \rho} \Omega_{\nu \rho}\right) & =-\frac{1}{4} R_{\mu \nu} \operatorname{Tr}\left(\gamma_{a b} \gamma_{c d}\right) R_{\mu \rho}{ }^{a b} R_{\nu \rho}{ }^{c d} \\
& =\frac{1}{2} R_{\mu \nu} R_{\mu \rho}{ }^{a b} R_{\nu \rho a b} \operatorname{Tr}(1) \\
& =\frac{1}{2}(2) g_{i j}\left(g_{i p} g_{m q}-g_{i q} g_{m p}\right)\left(g_{j p} g_{m q}-g_{j q} g_{m p}\right) 4 a^{-6} \\
& =2(9-3) 4 a^{-6}=12 \cdot 4 a^{-6}
\end{aligned}
$$

$$
\begin{aligned}
5 R \operatorname{Tr}\left(\Omega_{\mu \nu}^{2}\right) & =\frac{5}{16} R \operatorname{Tr}\left(\gamma_{a b} \gamma_{c d}\right) R_{\mu \nu}{ }^{a b} R_{\mu \nu}{ }^{c d} \\
& =-\frac{5}{8} R R_{\mu \nu \rho \sigma}^{2} \operatorname{Tr}(1) \\
& =-\frac{5}{8}(6)(12) 4 a^{-6} \\
& =-45 \cdot 4 a^{-6}
\end{aligned}
$$




$$
\begin{aligned}
& 60 \operatorname{Tr}\left(E^{3}\right)=60\left(-\frac{1}{4}\right)^{3} R^{3} \operatorname{Tr}(1) \\
& =60\left(-\frac{1}{4}\right)^{3}(6)^{3} \cdot 4 a^{-6} \\
& =-\frac{1}{2}(15 \cdot 27) \cdot 4 a^{-6} \\
& 30 \operatorname{Tr}\left(E \Omega_{\mu \nu}^{2}\right)=30\left(-\frac{3}{2}\right)(-2)\left(-\frac{1}{4}\right)^{2} a^{-2} R_{\mu \nu \rho \sigma}^{2} \operatorname{Tr}(1) \\
& =\frac{90}{16}(12) 4 a^{-6} \\
& =\frac{1}{2}(5 \cdot 27) \cdot 4 a^{-6} \\
& 30 R E^{2} \operatorname{Tr}(1)=\frac{30}{16} R^{3} \operatorname{Tr}(1) \\
& =\left(\frac{15}{8}\right)(6)^{3} 4 a^{-6} \\
& =(15 \cdot 27) \cdot 4 a^{-6} \\
& 5 R^{2} E \operatorname{Tr}(1)=-\frac{5}{4} R^{3} \cdot 4 \\
& =-\frac{5}{4}(6)^{3} 4 a^{-6} \\
& =-(10 \cdot 27) \cdot 4 a^{-6} \\
& -2 R_{\mu \nu}^{2} E \operatorname{Tr}(1)=-2 R_{\mu \nu}^{2}\left(-\frac{R}{4}\right) 4 \\
& =-2(12)\left(-\frac{3}{2}\right) 4 a^{-6} \\
& =36 \cdot 4 a^{-6} \\
& 2 R_{\mu \nu \rho \sigma}^{2} E \operatorname{Tr}(1)=2(12)\left(-\frac{3}{2}\right) 4 a^{-6} \\
& =-36 \cdot 4 a^{-6}
\end{aligned}
$$

Collecting the second set of terms we get

$$
\begin{aligned}
- & \frac{4 a^{-6}}{360}\left(9+18-12+45+\frac{15 \cdot 27}{2}-\frac{5 \cdot 27}{2}-15 \cdot 27+10 \cdot 27-36+36\right) \\
& =-\frac{2}{3} a^{-6}
\end{aligned}
$$

Thus the sum of all the terms in $a_{6}$ is zero. 


\section{Acknowledgements}

The research of A. H. C. is supported in part by the Arab Fund for Social

and Economic Development.

\section{REFERENCES}

[1] J. Milnor, Eigenvalues of the Laplace operator on certain manifolds Proc. Natl. Acad. Sci. U S A. 51(4) (1964), 542.

[2] A. Connes, On the spectral characterization of manifolds, to appear.

[3] A. Connes, Gravity coupled with matter and the foundation of noncommutative geometry, Comm. Math. Phys. 182 (1996) 155-176.

[4] John Barrett, The Lorentzian Version of the Noncommutative Geometry Model of Particle Physics, J. Math. Phys. 48: 012303 (2007).

[5] A. Connes, Noncommutative geometry and the standard model with neutrino mixing, JHEP 0611:081 (2006)

[6] A. Chamseddine and A. Connes, Why the Standard Model, Jour. Geom. Phys. 58 (2008) 38-47.

[7] A. Chamseddine and A. Connes, Conceptual explanation for the algebra in the noncommutative approach to the standard model, Phys. Rev. Lett. 99 (2007) 191601.

[8] A. Chamseddine and A. Connes, The Spectral action principle, Comm. Math. Phys. 186 (1997), 731-750.

[9] A. Chamseddine, A. Connes, M. Marcolli, Gravity and the standard model with neutrino mixing, Adv. Theor. Math. 11 (2007) 991-1090.

[10] A. Chamseddine and A. Connes, Scale invariance in the spectral action, Jour. Math. Phys. 47 (2006) 063504.

[11] A. Chamseddine and A. Connes, Quantum gravity boundary terms from the spectral action of noncommutative space, Phys. Rev. Lett. 99 (2007) 071302.

[12] G. Gibbons and S. Hawking, Action integrals and partition functions in quantum gravity, Phys. Rev. D 15 (1977) 2752.

[13] A. Ashtekar, J. Engle and D. Sloan, Asymptotics and Hamiltonians in a first order formalism, Class. Quant. Grav. 25 (2008) 095020.

[14] J. van der Bij, H. van Dam and Y. Ng, Physica A116 (1982) 307; F. Wilczek and A. Zee in High Energy Physics, ed. S. Mintz and A.Perlmutter, Plenum, NY, 1985; S. Weinberg, Rev. Mod. Phys. 61 (1989) 1.

[15] M. Reuter, Nonperturbative evolution equation for quantum gravity, Phys. Rev. D 57 (1998) 971.

[16] D. Dou, R. Percacci, The running gravitational couplings, Class. Quant. Grav. 15 (1998) 3449.

[17] R. Percacci, Renormalization group, systems of units and the hierarchy problem, J. Phys. A40 (2007) 4895.

[18] A. Chamseddine and A. Connes, Unocovering the noncommutative geometry of spacetime: a user manual for physicists, to appear.

[19] T. Hambye and K. Riesselmann, Matching Conditions and Upper bounds for Higgs Masses Revisited, Phys. Rev. D55: (1997) 7255.

[20] G. Isidori, V. Rychkov, A. Sturmia and N. Tetradis, Gravitational corrections to the standard model vacuum decay, Phys. Rev. D77 (2008) 025034.

[21] S. Coleman and E. Weinberg, Radiative corrections as the origin of spontaneous symmetry breaking, Phys. Rev. D7, (1973) 1888.

[22] W. Buchmüller and C. Busch, Symmetry breaking and mass bounds in the standard model with hidden scale invariance, Nucl. Phys. B 349 (1991) 71.

[23] P. Gilkey, Invariance Theory, the Heat Equation and the Atiyah-Singer Index Theorem, Wilmington, Publish or Perish, 1984. 
[24] H. B. Lawson, M-L. Michelsohn Spin geometry, Princeton Mathematical Series, 38. Princeton University Press, Princeton, NJ, 1989.

[25] S. Weinberg, Gravitation and Cosmology, J. Wiley, (1972) pages 389-390.

[26] A. Trautman, Spin structures on hypersurfaces and spectrum of Dirac ooperators on spheres, in Spinors, Twistors, Clifford Algebras and Quantum Deformations, Kluver Academic Publishers 1993.

[27] R. Camporesi and A. Higuchi, On the eigenfunctions of Dirac operators on spheres and hyperbolic spaces, J. Geom. Phys. 20 (1996) 1.

E-mail address: chams@aub.edu.1b

E-mail address: alain@connes.org 